\title{
Die Münzsammlung des Johann Christoph Olearius und das niederösterreichische Benediktinerstift Göttweig
}

\author{
von \\ MANUELA MAYER
}

Die eigene Hausgeschichte war und ist für Klöster, ungeachtet ihrer Ordenszugehörigkeit oder dem Geschlecht der Ordensleute, ein bedeutender Aspekt zur Identitätsstiftung, der meist seit der Klostergründung sorgsam gepflegt wird, wovon bis in die heutige Zeit zahlreiche (oft hauseigene) Publikationen zeugen. ${ }^{1} \mathrm{Zu}$ vielen Aspekten bieten diese Publikationen die bisher einzig verfügbare Auswertung. Ein vermehrtes Interesse externer Forscher könnte für die Zukunft dazu beitragen, neue Blickwinkel in altbekannte Thematiken einzubringen und zusätzliche Anknüpfungspunkte zu setzen, die aus einer rein lokalen Perspektive heraus nicht möglich wären. Wenngleich es bereits einen nicht zu unterschätzenden Prozentsatz an Personen ohne monastischen Hintergrund gibt, die sich um eine wissenschaftliche Aufarbeitung klosterbezogener Themen bemühen, überwiegt bis heute doch der Anteil interner Autoren, ohne deren Bemühungen freilich der Kenntnisstand um die Geschichte der Klöster weitaus geringer wäre. Meist sind es ihre Forschungsergebnisse, die verstärkt rezipiert und tradiert werden und durch die Öffnung vieler Klöster für den Tourismus eine beständige Verbreitung und Rezeption auch nach außen erfahren.

Im niederösterreichischen Benediktinerkloster Göttweig, etwa 80 Kilometer von Wien entfernt, steht vor allem der 50. Abt des Hauses, Gottfried Bessel (16721749), im Fokus. Dieser initiierte den barocken Neubau des Klosters, nachdem die alte Anlage 1718 durch einen Großbrand weitläufig zerstört worden war. Es ist

1 Es würde den Rahmen dieser Publikation sprengen, sämtliche Hausgeschichten von Klöstern, gedruckt wie ungedruckt, hier aufzuzählen. Gleichsam könnte eine exemplarische Nennung ihrer Anzahl, Qualität und unterschiedlichen Zielsetzung nicht gerecht werden. Für den deutschsprachigen Raum sei für die Benediktiner auf die Reihe "Germania Benedictina“ (12 Bde., St. Ottilien 1970-2014) sowie für die AugustinerChorherren auf die Reihe „Österreichisches Chorherrenbuch“ (3 Bde., Klosterneuburg/ Wien 1994-2005; miteinbezogen wurden auch Klöster in Südtirol, Böhmen, Mähren und Ungarn) verwiesen. Beide Reihen bieten eine wissenschaftliche Aufarbeitung von Hausgeschichten der behandelten Klöster nebst umfangreicher Bibliografie. Darüber hinaus haben zahlreiche Orden wissenschaftliche Zeitschriften ins Leben gerufen, in denen immer wieder hausgeschichtliche Aspekte aufgegriffen werden, etwa "Studien und Mitteilungen zur Geschichte des Benediktinerordens und seiner Zweige“, „Analecta Cartusiana“ oder „Analecta Cisterciensia“. 
deshalb nicht verwunderlich, dass die Baugeschichte breiten Raum in der bisherigen Besselforschung und -memoria eingenommen hat. ${ }^{2}$ Weitere viel zitierte Sujets sind Bessels Verdienste um den Ausbau von Stiftsbibliothek und Sammlungen ${ }^{3}$ sowie sein Beitrag zu den Historischen Hilfswissenschaften. ${ }^{4}$

Eine vor allem von den Archivaren, Bibliothekaren und Kuratoren des Stiftes getragene rege Publikationstätigkeit hält die Würdigung Abt Bessels bis heute aufrecht. Dabei trug sie nicht nur zur beständigen Wiederholung mancher Angaben in der Literatur bei, sondern auch zu deren allgemeinen Akzeptanz. So gehört es etwa zur immer noch gültigen Fama des Münzkabinetts, dass dessen Umfang mit 25000 Stück angegeben wird, bestehend aus 10000 antiken, 11000 mittelalterlichen und neuzeitlichen Münzen sowie 4000 Medaillen. ${ }^{5}$ Schon Wolfgang

2 Vgl. Gregor Martin Lechner, Göttweig, in: Ulrich Faust/Waltraud Krassnig (Hg.), Germania Benedictina, Bd. 3/1: Die benediktinischen Mönchs- und Nonnenklöster in Österreich und Südtirol, St. Ottilien 2000, S. 768-843, hier S. 783 f.; Gregor MarTin Lechner/Michael Grünwald, Gottfried Bessel (1672-1749) und das barocke Göttweig. Zum 250. Todesjahr des Abtes. Ausstellung des Archivs und der Sammlungen des Stiftes Göttweig/Niederösterreich, 24. April bis 15. November 1999, Furth bei Göttweig 1999, S. 108-111; Emmeram RitTER, Gottfried Bessel als Bauherr und Kunstmäzen, in: Franz Rudolf Reichert (Hg.), Gottfried Bessel (1672-1749). Diplomat in Kurmainz - Abt von Göttweig - Wissenschaftler und Kunstmäzen (Quellen und Abhandlungen zur mittelrheinischen Kirchengeschichte 16), Mainz 1972, S. 93-140, hier S. 93-125; EdMund VAšıčEK, Abt Gottfried von Bessel von Göttweig. Ein Lebensbild, Wien 1912, S. 95-102.

3 Vgl. Lechner, Göttweig (wie Anm. 2), S. 797-800, 811-814; Gregor Martin LechNER/Herbert Fasching, Stift Göttweig und seine Kunstschätze, St. Pölten/Wien 1977, S. 24 f., 75, 84-86; Lechner/Grünwald, Bessel und das barocke Göttweig (wie Anm. 2), S. 112-115; Emmeram Ritter, Geschichte der graphischen Sammlung des Stiftes Göttweig, in: Ostbairische Grenzmarken 11 (1969), S. 249-277; Ders., Gottfried Bessel - der „deutsche Mabillon“, in: Reichert, Gottfried Bessel (wie Anm. 2), S. 203-215, hier S. 206-208; RitTer, Bauherr (wie Anm. 2), S. 125-137; VAšıčEK, Bessel (wie Anm. 2), S. 143-146.

4 Bessel plante eine dreibändige, quellenbasierte Hausgeschichte (!) mit dem Titel „Chronicon Gotwicense“, von der 1732 jedoch nur der erste Band mit einer Einführung in mittelalterliche Handschriften- und Urkundenlehre erschienen ist. Trotz des fragmentarischen Charakters brachte dieser Band Bessel den noch von seinen Zeitgenossen vergebenen Ruf eines „deutschen Mabillon“ ein, da er unter Verwendung der von Jean Mabillon (1632-1707) begründeten kritischen Urkundenlehre das erste Handbuch dieser Art für den österreichischen Raum vorgelegt hatte. Vgl. Peter Albert, Gottfried Bessel und das Chronicon Gottwicense, in: Freiburger Diöcesan-Archiv 27 (1899), S. 217-250; Lechner/Grünwald, Bessel und das barocke Göttweig (wie Anm. 2), S. 82-85; Ritter, Mabillon (wie Anm. 3), S. 210-215; Peter G. Tropper, Urkundenlehre in Österreich vom frühen 18. Jahrhundert bis zur Errichtung der „Schule für Österreichische Geschichtsforschung “ 1854 (Publikationen aus dem Archiv der Universität Graz 28), Graz 1994, S. 26-46; VAšıčEK, Bessel (wie Anm. 2), S. 118-141.

5 Vgl. Gregor Martin Lechner, Einleitung, in: Wolfgang Szaivert, Die Münzsammlung des Benediktinerstiftes Göttweig (Veröffentlichungen der numismatischen Kommission 12; Thesaurus nummorum Romanorum et Byzantinorum 5) Wien 1983, S. 19-25, hier S. 24; Lechner, Göttweig (wie Anm. 2), S. 812; Lechner/Fasching, Kunstschätze (wie Anm. 3), S. 26; Lechner/Grünwald, Bessel und das barocke Göttweig (wie Anm. 2), S. 113; RitTer, Bauherr (wie Anm. 2), S. 131. 
Szaivert kommt in seiner 1983 erschienenen Bearbeitung der antiken Münzen der Göttweiger Sammlung hingegen auf eine Maximalzahl für diese Untergruppe von 8137 Stück. ${ }^{6}$ Wie Stefan Spevak vor einigen Jahren zeigen konnte, benennen die kolportierten Zahlen den Umfang des Bestandes vor der Aufhebung des Klosters im Jahr 1939.7 Da bekanntlich, auch dies ist fixer Bestandteil der modernen Hausgeschichte, nicht alle beschlagnahmten Objekte nach der Wiedererrichtung des Klosters restituiert werden konnten und zudem eine Aufarbeitung der übrigen Bestände des Münzkabinetts noch ausständig ist, kann der aktuelle Umfang der Sammlung nur geschätzt werden. Dennoch halten sich die oben genannten Zahlen bis heute in der Literatur.

Etwas treffsicherer verhält es sich mit der Kenntnis um die Provenienz von Konvoluten und Privatsammlungen, mit denen das Göttweiger Münzkabinett unter Abt Bessel vermehrt wurde, wenngleich sich auch hier die eine oder andere Unschärfe in den Angaben entdecken lässt. So gehört es zum bisherigen Konsens, dass Bessel, neben kleineren Münzkäufen sowie einer Erbschaft der Sammlung seines 1724 verstorbenen Bruders Johann Franz (1683-1724), die Münzsammlungen von Johann Christoph Olearius (1668-1747), Christian Schlegel (1667-1722) und Konrad Sigler ( $†$ 1723) erwarb. ${ }^{8}$ Bezüglich der Sammlung Schlegel merkte Gregor Martin Lechner 1983 an, dass es in diesem Fall möglicherweise zu keinem Vertragsschluss gekommen sein könnte. ${ }^{9}$ Wenngleich sich diese Vermutung, wie im Folgenden gezeigt werden wird, als richtig erwiesen hat, ist sie in der Literatur nicht weiter aufgegriffen worden.

Die Herkunft der Göttweiger Münzbestände war also niemals ein Geheimnis und wurde sogar mehrfach publiziert. Dennoch hat, mit Ausnahme einiger weniger Numismatiker aus dem Umkreis des Instituts für Numismatik und Geldgeschichte der Universität Wien wie Robert Göbl oder Wolfgang Szaivert, die Fachwelt kaum Notiz von dieser Information genommen. Dies mag einerseits der geringen Reichweite der Publikationen (zumeist Göttweiger Eigenerzeugnisse anlässlich diverser Jubiläen) geschuldet sein, mag sich andererseits aber auch durch anders gewichtete Forschungsinteressen in der österreichischen Numismatik erklären lassen. In jedem Fall wurde damit die paradoxe Situation erschaffen, dass im

6 Vgl. Szaivert, Münzsammlung Göttweig (wie Anm. 5). Eine Bearbeitung der übrigen Münzen und Medaillen ist noch ausständig.

7 Vgl. Stefan Spevak, Kunst im Benediktinerstift Göttweig. Entzug unter der NS-Herrschaft und Restitution nach 1945, in: Verena Pawlowsky/Harald Wendelin (Hg.), Enteignete Kunst. Raub und Rückgabe in Österreich von 1938 bis heute, Wien/Berlin 2006, S. 142-157, hier S. 146.

8 Vgl. Robert Göвl, Das Göttweiger Münzkabinett und die Ausstellung „Mundus Christianus in nummis“, in: 900 Jahre Stift Göttweig 1083-1983. Ein Donaustift als Repräsentant benediktinischer Kultur, Göttweig 1983, S. 160-237, hier S. 161; LECHNER, Einleitung (wie Anm. 5), S. 19 f.; Lechner, Göttweig (wie Anm. 2), S. 812; Lechner/ Fasching, Kunstschätze (wie Anm. 3), S. 26; Lechner/Grünwald, Bessel und das barocke Göttweig (wie Anm. 2), S. 113; RitTer, Bauherr (wie Anm. 2), S. 133.

9 Vgl. Lechner, Einleitung (wie Anm. 5), S. 19. 
Umkreis der Besselforschung die genannten Ankäufe zwar wohlbekannt waren, ihre Bedeutung aber konsequent unterschätzt wurde, wohingegen in Gotha, Arnstadt und Erfurt, den Zentren der Oleariusforschung mit ihren zahlreichen verdienstvollen VertreterInnen, der Verbleib der Münzsammlung des Johann Christoph Olearius aber unbekannt blieb.

Der folgende Beitrag möchte die entstandene Lücke zwischen den bisherigen Forschungen zu Gottfried Bessel und Johann Christoph Olearius schließen und dazu beitragen, die Ergebnisse jenen Fachleuten zugänglich zu machen, für deren Forschungsbereiche sie von Interesse sind.

\section{Der Neubau des Stiftes und seine Auswirkungen auf die Stiftssammlungen}

Dass Gottfried Bessel überhaupt an einer Erweiterung der Göttweiger Stiftssammlungen gelegen war, hatte einen unangenehmen Hintergrund: am Abend des 17. Juni 1718 war im Stift Göttweig ein Brand ausgebrochen, der mangels ausreichender Wasserversorgung nicht gelöscht werden konnte und somit unaufhaltsam die gesamte Klosteranlage zerstörte. Allerdings breitete sich das Feuer langsam genug aus, um die Bestände von Bibliothek, Archiv und Sammlungen zu retten. ${ }^{10}$ Was folgte, war notwendigerweise der komplette Neubau der Klosteranlage, mit dem der kaiserliche Hofarchitekt Johann Lukas von Hildebrandt (1668-1745) beauftragt wurde, dessen Entwürfe sich gegen jene von Jakob Prandtauer (16601726) und Balthasar Neumann (1687-1753) durchsetzten. Die Bauleitung übernahm schon 1722 Hildebrandts Mitarbeiter Franz Jänggl (1650-1734), der Hildebrandt ab 1725 auch als Architekt beerbte, da sich dieser aus gesundheitlichen Gründen aus dem Göttweiger Auftrag zurückzog. Nach dem Tod Jänggls führte dessen Neffe Franz Anton Pilgram (1699-1761) die Bauarbeiten fort. ${ }^{11}$

Bessel erlebte die Fertigstellung des Neubaus nicht mehr, wiewohl aber ein Großteil des Konzepts in seiner Amtszeit umgesetzt werden konnte. Nach der Grundsteinlegung am 2. Juli 1719 wurde als erstes mit dem Osttrakt begonnen, der die Räumlichkeiten für die Konventualen beherbergte, sodass diese bereits im November 1724 nach Göttweig zurückkehren konnten. Bis 1727 folgte die Fertig-

10 Die geretteten Mobilien wurden bis zur Wiedererrichtung des Klosters auf zum Stift gehörige Pfarren und Gutshöfe verbracht. Die Konventualen wurden auf mehrere andere österreichische Benediktinerklöster verteilt. Vgl. Lechner, Göttweig (wie Anm. 2), S. 783; Lechner/Grünwald, Bessel und das barocke Göttweig (wie Anm. 2), S. 108; RitTer, Bauherr (wie Anm. 2), S. 94; VAšıčeK, Bessel (wie Anm. 2), S. 95-97.

11 Vgl. Lechner, Göttweig (wie Anm. 2), S. 783; Lechner/Grünwald, Bessel und das barocke Göttweig (wie Anm. 2), S. 108 f.; RitTER, Bauherr (wie Anm. 2), S. 95-100, 104109; Emmeram Ritter, Der NÖ. Landschaftsbaumeister Franz Anton Pilgram, in: Unsere Heimat. Zeitschrift des Vereines für Landeskunde von Niederösterreich und Wien 34 (1963), S. 114-123; PÁl VoIT, Unbekannte Pläne Johann Lucas von Hildebrandts und der Neuaufbau des Stiftes in Göttweig 1719-1746, in: Wiener Jahrbuch für Kunstgeschichte 28 (1975), S. 123-144, hier S. 123-126. 
stellung der Prälatur, des Winterrefektoriums, der Werkstätten und einiger Verwaltungsgebäude, ehe die Bautätigkeit für einige Jahre, bis auf kleinere Arbeiten, fast eingestellt wurde. Gründe dafür waren der Wechsel in der Bauleitung, Schwierigkeiten in der Materialbeschaffung sowie Finanzierungsprobleme. 1733 wurde mit dem Bau der Stiftskirche und des Gästetraktes begonnen. Zudem wurden in dieser Bauphase die Dachstühle für alle Gebäude fertiggestellt. Bei Bessels Tod im Jahr 1749 befand sich die Fassade der Stiftskirche noch in Arbeit und auch der Westtrakt mit weiteren Verwaltungsgebäuden war noch nicht fertiggestellt. ${ }^{12}$

$\mathrm{Zu}$ jenen Gebäudeteilen, die als erstes gebaut und fertiggestellt wurden, da sie für die Konventualen wichtig waren, zählte auch der sogenannte Frauenturm in der nordöstlichen Ecke der Klosteranlage, in dem die Stiftssammlungen untergebracht werden sollten. ${ }^{13}$ Dieser Turm hätte ursprünglich bereits 1722 fertiggestellt werden sollen, jedoch hatte sich Bessel dazu entschlossen, sämtliche Türme um ein Stockwerk zu erhöhen, sodass der „Frauenturm“ letztlich erst 1723 eröffnet werden konnte. ${ }^{14}$ Gemeinsam mit Bibliothek (ebenfalls im Osttrakt) und Archiv (angrenzend im Nordtrakt) waren die Sammlungen ein unverzichtbarer Baustein für die Ausbildung der Novizen in der klostereigenen Hauslehranstalt. ${ }^{15}$ Neben diesem pädagogischen Aspekt verfügten die Stiftssammlungen natürlich auch über einen stark repräsentativen Charakter. Dieser wurde deutlich, wenn Gäste durch die Sammlungen geführt wurden, bei denen es sich gleichermaßen um Äbte und Konventualen anderer Klöster handeln konnte wie um adlige Reisende, die das Kloster für einen Zwischenstopp nutzten. Doch auch hausinterne Forschungen sollten durch die Sammlungen unterstützt und gefördert werden. ${ }^{16}$

12 Vgl. Lechner, Göttweig (wie Anm. 2), S. 808-810; Lechner/Grünwald, Bessel und das barocke Göttweig (wie Anm. 2), S. 108-111; RitTer, Bauherr (wie Anm. 2), S. 109113; RITTER, Landschaftsbaumeister (wie Anm. 11), S. 116; VAŠIČEK, Bessel (wie Anm. 2), S. 99-102.

13 Emmeram Ritter lokalisierte die Sammlungen zur Zeit Abt Bessels irrig im südöstlichen „Altmanniturm“; vgl. RitTer, Bauherr (wie Anm. 2), S. 128; Ders., Geschichte (wie Anm. 3), S. 265. In diesen Turm wurden sie aber erst im 19. Jahrhundert transferiert. Dank für diese Information an Bernhard Rameder, Stift Göttweig.

$14 \mathrm{Vgl}$. Lechner/Grünwald, Bessel und das barocke Göttweig (wie Anm. 2), S. 109; RitTer, Bauherr (wie Anm. 2), S. 110.

15 Ihre vollständige pädagogische Bedeutung erhielten die Sammlungen durch weitere Maßnahmen Abt Bessels, etwa die Gründung einer Trivialschule, in der sämtliche Kinder der Göttweiger Grundherrschaft per verordneter Schulpflicht kostenlos unterrichtet wurden, oder die geplante Gründung einer neuen Schulform für die Sekundarstufe, die sowohl Elemente der Ritterakademien als auch der Gymnasien in sich vereinen sollte. Vgl. Helmut Engelbrecht, Geschichte des österreichischen Bildungswesens. Erziehung und Unterricht auf dem Boden Österreichs, Bd. 3: Von der frühen Aufklärung bis zum Vormärz, Wien 1981, S. 17-19; Ders., Gottfried Bessels Beitrag zum pädagogischen Umdenken seiner Zeit, in: Reichert, Gottfried Bessel (wie Anm. 2), S. 173-202, hier S. 181-193; Ludwig Koller, Errichtung der Göttweiger Trivialschule, in: Monatsblatt des Altertums-Vereines zu Wien 10 (1911), S. $45 \mathrm{f}$.

16 Zum breiten Spektrum klösterlicher Sammlungen vgl. Georg SсHRotT, Klösterliche Sammelpraxis in der Frühen Neuzeit. Typologie, Geschichte, Funktionen und Deutungen, in: Georg Schrott/Manfred Knedlik (Hg.), Klösterliche Sammelpraxis in der 
Im Fall der Göttweiger Sammlungen, deren Ursprung bis ins 16. Jahrhundert auf die Sammeltätigkeit einzelner Äbte sowie Stiftungen von Gläubigen zurückgeht, ist zu beobachten, dass sich der Anspruch der Repräsentation und der Wissenschaftlichkeit auch in der Struktur und der Präsentation der Sammlungen niederschlug. War die alte Sammlung (also vor dem Brand), über deren Systematik und Aufbewahrungsort kaum etwas bekannt ist, am ehesten mit einer Kunst- und Wunderkammer gleichzusetzen, so galt dieses Konzept zu Beginn der Aufklärung als veraltet. Stattdessen dominierten nun genrespezifische Spezialsammlungen, in denen die Objekte nach Themengebieten oder Funktionen geordnet wurden. ${ }^{17}$

Der Umstand, dass die Sammlungen mit der Eröffnung des „Frauenturms“ in zwei eigens dafür konzipierte Räume von adäquater Größe einziehen konnten, bot gleichzeitig die Gelegenheit für eine Umstrukturierung des Objektbestandes. Das Ergebnis wurde 1744 vom Kupferstecher Salomon Kleiner (1700-1761) festgehalten, der in einem größeren Auftrag mit Ansichten der bereits fertiggestellten Stiftsgebäude, der Bibliothekssäle und der Sammlungen betraut worden war.

Gemäß Kleiners Darstellungen wurden auch nach der Neuaufstellung der Sammlungen Objekte unterschiedlicher Genera gemeinsam präsentiert, jedoch waren sie nun zu thematischen Untergruppen zusammengefasst. So enthielt der obere der beiden Ausstellungsräume, von Kleiner „Musaei contignatio superior“ betitelt, Grafiken, Waffen, Jagdtrophäen, präparierte Vögel sowie überlange Objekte, etwa Speere, Schwertfische, Narwalzähne und präparierte Schlangen. ${ }^{18}$ Der andere Ausstellungsraum, von Kleiner als „Musaei contignatio media“ bezeichnet, da es sich um das mittlere von drei Turmzimmern handelte, beherbergte Fossilien, in Alkohol eingelegte Tierkörper, menschliche Präparate, Münzen, Kunstgegenstände und Antiken. ${ }^{19}$ Nicht dargestellt werden konnte die reiche Gemäldesammlung, da deren Bestand zur Erbauung der Konventualen im gesamten Kloster verteilt ausgestellt war.

Wiewohl sich Kleiners Darstellungen durch eine große Detailtreue auszeichnen, darf nicht vergessen werden, dass es sich dabei auch um eine künstlerische Darstellung handelt, bei deren Umsetzung Kleiner auf zahlreiche Vorbilder zurückgreifen konnte. Abbildungen von Sammlungen wurden bis zur Mitte des 18. Jahrhunderts derart beliebt, dass sie sich zu einem eigenständigen, mit eigenen stilistischen Merkmalen ausgestatteten Bildtypus entwickelten. So wurde der jeweilige Ausstellungsraum stets in seiner Gesamtheit vom Boden bis zur Decke gezeigt, damit auch die sich darin befindlichen Vitrinen mit all ihren Objekten in

Frühen Neuzeit (Religionsgeschichte der Frühen Neuzeit 9), Nordhausen 2010, S. 7-71; Klaudius Wintz, Die Kunst- und Wunderkammer, in: Gerfried Sitar/Martin Kroker/ Holger Kempkens (Hg.), Macht des Wortes - Benediktinisches Mönchtum im Spiegel Europas, Regensburg 2009, S. 385-393, hier S. 391.

17 Vgl. Schrott, Sammelpraxis (wie Anm. 16), S. 21-24; Wintz, Wunderkammer (wie Anm. 16), S. 388-390.

18 Graphische Sammlung Stift Göttweig, Hg_013.

19 Ebd., Hg_012. 
voller Größe gezeigt werden konnten. Ein Fluchtpunkt in der Mitte des Bildes machte es möglich, auch die Seitenwände des Raumes in das Bild zu integrieren, sodass sich für den Betrachter der Raum wie ein Bühnenbild öffnete. Ein Element im Zentrum des Bildes, etwa ein Möbelstück mit darauf drapierten Sammlungsobjekten, lenkte den Fokus der Aufmerksamkeit von der Umgebung zurück auf die Sammlung selbst und suggerierte dabei gleichzeitig, dass es sich nicht nur um eine museale Ausstellung handelte, sondern auch um eine wissenschaftliche Forschungsstätte. ${ }^{20}$

Es zählt zu den Meriten Kleiners, die künstlerischen Attribute dieses Bildtypus mit realen Elementen aus den Göttweiger Sammlungen zu einem Idealbild zu verweben, in dem sämtliche der vorhandenen Spezialsammlungen vertreten waren. Der repräsentative Charakter speiste sich dabei aber weniger aus einer quantitativen Fülle von Objekten, sondern überzeugte durch die sorgsame Zusammenstellung ausgewählter Stücke. ${ }^{21}$ Dass Kleiner dabei Objekte aus den Sammlungen als Vorlage für seine Komposition verwendete, zeigt sich aus dem Umstand, dass sich zahlreiche der von ihm dargestellten Gegenstände noch heute in den Sammlungen nachweisen lassen. Dazu zählen auch die abgebildeten Münzschränke als Repräsentanten des Münzkabinetts. Von den vier Münzschränken und einem u-förmigen Tisch mit Münzladen haben sich noch zwei Schränke in der aktuellen Sammlung erhalten. Der Tisch wird zuletzt in einem Inventar aus dem Jahr 1867 erwähnt.22

$\mathrm{Zu}$ der Zeit, als Kleiner mit der Darstellung der Sammlungen beauftragt wurde, waren deren Bestände, zumindest nach Bessels Konzeption, bereits zu großen Teilen konsolidiert. Damit es aber so weit kommen konnte, hatte Bessel unmittelbar nach der Fertigstellung der Sammlungsräume und der Eröffnung des "Frauenturms“ eine äußerst aktive Ankaufspolitik initiiert, welche die quantitative Erweiterung der Bestände zum Ziel hatte, wobei aber gleichzeitig der Versuch unternommen wurde, möglichst interessante und wertvolle Stücke zu erwerben.

Bezogen auf die Münzsammlung ist zu bemerken, dass Bessel dabei kaum Wert auf antike Münzen legte, was gemeinhin mit seinem persönlichen (Forschungs-) Interesse für mittelalterliche Geschichte argumentiert wird. In einem heute verschollenen Brief vom 25. April 1725 an Johann Gottfried Weribert von Person (fl. 1719-1725), einen Mitarbeiter Johann Lukas von Hildebrandts, spezifizierte Bessel, welche Münzen er für das Göttweiger Münzkabinett vorrangig anzukaufen gedachte, nämlich Münzen deutscher Kaiser des Mittelalters bis auf Maximilian I.

20 Vgl. Schrotт, Sammelpraxis (wie Anm. 16), S. $41 \mathrm{f}$.

21 Zahlreiche schriftliche Quellen, wie etwa Berichte von Besuchern der Sammlungen, aber auch Teilinventare, legen nahe, dass die Sammlungen weit umfangreicher waren, als von Kleiner dargestellt.

22 Vgl. Göвl, Münzkabinett (wie Anm. 8), S. 163; Lechner, Einleitung (wie Anm. 5), S. 24. 
(1459-1519).23 Bessel bat Person, sich im Umfeld der Städte Breisach, Straßburg und Freiburg nach solchen Münzen umzusehen. Weiters bekundete er auch sein Interesse an Brakteaten, jedoch nur an solchen, die auch Inschriften aufwiesen.24

Diese kurze Instruktion macht bereits deutlich, dass Bessel bei der quantitativen Erweiterung der Göttweiger Stiftssammlungen auf die Hilfe und Vermittlung Dritter baute. Dies ist nicht weiter verwunderlich, waren ihm als Abt eines benediktinischen Klosters, für das die stabilitas loci galt, räumliche Grenzen gesetzt, die durch die geografische Lage des Stiftes in der Peripherie weiter eingeschränkt wurden. Zudem übte Bessel parallel zu seinem Abbatiat mehrere Ämter aus, die seine Aufmerksamkeit forderten: so war er zweifacher Rektor der Universität Wien in den akademischen Jahren 1714/15 und 1726/27, Verordneter im Prälatenstand des Niederösterreichischen Landtags und mehrfach im Auftrag Kaiser Karls VI. (1685-1740) als Diplomat tätig. Letztlich forderte auch der Klosterneubau eine intensive Bindung an Göttweig ein. ${ }^{25}$

\section{Der Ankauf der Sammlung Olearius}

Im erhaltenen Nachlass Bessels füllen die Korrespondenzen mit seinen Agenten, die für ihn Bücher und Objekte ankauften, zwei Sammelhandschriften. ${ }^{26}$ Eine Analyse dieser Korrespondenzen zeigt, dass Bessel hier vor allem auf Vertraute und Familienangehörige setzte: neben dem bereits erwähnten Johann Gottfried Weribert von Person, dessen Bekanntschaft Bessel wohl im Zuge der Planungen für den Stiftsneubau gemacht hatte, zählte sein in Rom lebender Bruder Johann Franz ebenso zu seinen Agenten wie sein Schwager Johann Michael Bockleth († vor 1747) in Erfurt und dessen Bruder Georg Joseph Bockleth (1685-1731) in Würzburg. Während Bessel seinen Bruder vor allem auf Bücher italienischer Drucker ansetzte, die in Wien nur schwer oder gar nicht zu bekommen waren,

23 Vgl. Göвl, Münzkabinett (wie Anm. 8), S. 161; Lechner, Einleitung (wie Anm. 5), S. 21; RitTer, Bauherr (wie Anm. 2), S. 132 f. Die bei Ritter angegebene Archivsignatur lässt sich nicht verifizieren. Dank für diese Information an P. Franz Schuster, Stift Göttweig.

24 Es liegt die Vermutung nahe, dass sich Person zur Zeit dieses Briefes im genannten Raum aufhielt oder aber über Kontakte dorthin verfügte. Da über ihn aber so gut wie nichts bekannt ist, lässt sich diese Vermutung nicht verifizieren.

25 Stellvertretend für die kaum überschaubare Anzahl an Biografien bzw. biografischen Abrissen Bessels sei verwiesen auf Clemens Anton Lashofer, Profeßbuch des Benediktinerstiftes Göttweig. Zur 900-Jahr-Feier der Gründung des Klosters (Studien und Mitteilungen zur Geschichte des Benediktinerordens und seiner Zweige. Ergänzungsbände 26), St. Ottilien, 1983, S. 190, 205-209; Emmeram RitTER, Gedächtnisausstellung zur Wiederkehr des 300. Geburtstages von Abt Gottfried Bessel 1672-1749. Politiker, Gelehrter und Mäzen des Barock, Göttweig 1972, S. 8-13; Peter G. Tropper, Abt Gottfried Bessel (1714-1749), in: 900 Jahre Stift Göttweig (wie Anm. 8), S. 644-678; VAšıčEK, Bessel (wie Anm. 2).

26 Stiftsbibliothek Göttweig, Cod. 691, 692. 
versorgten die Brüder Bockleth ihn vor allem mit Fossilien, optischen Geräten, Antiken, Münzen und Spezialliteratur. Bezüglich der Münzen gelang es ihnen sogar, die umfangreichsten Privatsammlungen zu erwerben, die in der Geschichte der Göttweiger Sammlungen angekauft wurden und deren Vorbesitzer renommierte Mitglieder der res publica litteraria waren.

Obwohl die Namen von Bessels Agenten in der so zahlreich vorhandenen Literatur zu den Göttweiger Sammlungen beständig wiederholt wurden, war dennoch nur wenig über sie bekannt. ${ }^{27}$ Zusätzliche Informationen aus bisher nicht berücksichtigten Archivquellen liefern wertvolle Hinweise, die erstmals Aussagen über die vielfachen sozialen Verflechtungen erlauben. So hatte Bessels Bruder Johann Franz in Erfurt und Rom studiert, ehe er auf Vermittlung seines Bruders Hofkaplan des Mainzer Erzbischofs und Kurfürsten Lothar Franz von Schönborn (1655-1729) wurde, für den er als Mainzer Agent in Rom tätig war. Durch Kardinal Michael Friedrich von Althan (1680-1734) wurde er schließlich zum kaiserlichen Agenten in Rom und kaiserlichen Archivar ernannt. ${ }^{28}$

Der Erfurter Johann Michael Bockleth war mit Bessels Schwester Maria Eva (1680-nach 1747) verheiratet. In den Steuerbüchern der Stadt Erfurt wird er für die Jahre 1718 und 1734 als kurfürstlicher Kammerrat, Obristratsmeister, kaiserlicher Rat und Biereige geführt. Die Familie führte das Gasthaus „Zur hohen Lilie“ am Domplatz. Wann Bockleth verstarb, ist unklar. Im nächsten Eintrag des Steuerbuches von 1747 wird nur noch seine Witwe genannt, die wiederum ein Gasthaus betrieb, nämlich jenes zur „Großen und kleinen Klette“ in der Pilse. ${ }^{29}$

Etwas besser ist die Quellenlage für seinen Bruder Georg Josef, der eine geistliche Laufbahn einschlug und nach seiner Priesterweihe Hofkaplan des Würzburger Fürstbischofs Johann Philipp von Greiffenclau zu Vollraths (1652-1719) und von dessen Nachfolger Johann Philipp Franz von Schönborn (1673-1724) zum Geistlichen Rat ernannt worden war. ${ }^{30}$

Es wird deutlich, dass alle drei Verwandte im Dienst der Reichsgrafen von Schönborn standen, und es ist davon auszugehen, dass Gottfried Bessel erheblichen Anteil an der Versorgung seiner Angehörigen hatte, stand er doch selbst von 1699 bis 1714 als Hofkaplan und Offizial im Dienst des Mainzer Erzbischofs und Kurfürsten Lothar Franz von Schönborn. Zu diesem hielt Bessel auch als Abt von

27 Vgl. Lechner, Göttweig (wie Anm. 2), S. 812; Lechner/Fasching, Kunstschätze (wie Anm. 3), S. 26; Lechner/Grünwald, Bessel und das barocke Göttweig (wie Anm. 2), S. 113; Ritter, Bauherr (wie Anm. 2), S. 133; VAšičEk, Bessel (wie Anm. 2), S. 147.

28 Vgl. Helmut Brosch, Die Familie Bessel, in: Reichert, Gottfried Bessel (wie Anm. 2), S. 1-24, hier S. 14 f.; FrITZ Wiegand, Namensverzeichnis zur allgemeinen Studentenmatrikel der Universität Erfurt für die Zeit von 1637 bis 1816, Erfurt 1966, S. 35.

29 Vgl. Brosch, Familie Bessel (wie Anm. 28), S. 12 f. Stadtarchiv Erfurt, Register der städtischen Steuerbücher (Datenbank, 15. Dezember 2017) nach: Verrechtsbücher der Stadt Erfurt, Stadtarchiv Erfurt 1-1/XXIIIa- Bände 82, 87, 97. Dank an Antje Bauer, Stadtarchiv Erfurt.

30 Diözesanarchiv Würzburg, Auszug aus der Klerikerdatenbank (6. März 2018). Dank an Thomas Wehner, Diözesanarchiv Würzburg. 
Göttweig Kontakt, zumal Schönborn sich für die Wahl Bessels eingesetzt hatte, und engagierte sich seinerseits vielfach in Belangen der Familie Schönborn, etwa der Wahl von Johann Philipp Franz zum Würzburger Fürstbischof. ${ }^{31}$

Dass Bessel im Verbund mit dem Mainzer Erzbischof seinen Bruder zum Agenten in Rom machte, wurde bereits erwähnt, doch auch die Position Johann Michael Bockleths als kurfürstlicher Kammerrat trägt diese Handschrift, da die Stadt Erfurt bekanntlich unter der Herrschaft des Kurfürstentums Mainz stand. Letztlich kann es auch kein Zufall sein, dass Georg Joseph Bockleth mit dem Amtsantritt eines Schönborn in Würzburg dort zum Geistlichen Rat ernannt wurde.

Wenn Bessel nun also seine Verwandten mit Kaufaufträgen ausstattete, dann war dies nicht nur ein auf familiären Beziehungen fußender Vertrauensbeweis, sondern auch die Aufforderung eines Patrons an seinen Günstling zum Gegendienst. Vor allem aus der Korrespondenz der Brüder Bockleth mit Bessel wird deutlich, dass beide Parteien einander formal als nicht ebenbürtig ansahen beziehungsweise ansehen durften, denn während Bessel von seinen Agenten konsequent als höhergestellte Person angesprochen wird, wählen sie für sich selbst durchgehend Devotionsformeln. ${ }^{32}$ In keinem einzigen Brief etwa wird Bessel von Johann Michael Bockleth als sein Schwager bezeichnet, als einziger Hinweis auf ein verwandtschaftliches Verhältnis werden gelegentlich Grüße der „Frau Schwester" bestellt. Doch während die Brüder Bockleth Bessel jene Ehre erweisen mussten, die sein Amt erforderte, konnte es sich Bessel andererseits leisten, den Bruder seines Schwagers einmal auch als „hochgeehrten Herrn Vetter" anzusprechen.33

Dass die Agenten trotz aller verwandtschaftlichen oder freundschaftlichen Verbindungen keinesfalls autonom im Namen Bessels auftreten und handeln durften, wird ebenfalls anhand der Korrespondenz deutlich. Diese zeichnet sich durch einen beständigen Informationsfluss aus, der vor allem von den Agenten getragen wird. Jede neue Möglichkeit zur Akquise von Objekten wird Bessel ebenso mitgeteilt wie aktuelle Wendungen in den Verhandlungen mit einem Verkäufer oder Besichtigungstermine von zum Verkauf stehenden Privatsammlungen. Zudem wurde vor jedem weiteren Schritt erst die Zustimmung Bessels eingeholt. Auf diese Weise behielt Bessel, dessen Rolle ansonsten passiv blieb, die Kontrolle über

31 Vgl. Friedhelm Jürgensmeier, Abt Gottfried Bessel und die Reichsgrafen von Schönborn, in: Reichert, Gottfried Bessel (wie Anm. 2), S. 53-74, bes. S. 63-65; Ders., Dr. Gottfried Bessel im Dienst der Reichsgrafen von Schönborn, in: ebd., S. 25-52, bes. S. 25-29.

32 Bessel wird meist als „hochwürdiger“, „hochgelehrter“, „hochedelgeborener gnädiger Herr" angesprochen, während die Brüder Bockleth für sich selbst Formulierungen wie "gehorsamster/untertänigster" „Diener/Knecht" verwenden.

33 Stiftsbibliothek Göttweig, Cod. 691, fol. 129r. Zu den Ausprägungen sozialer Hierarchien in Korrespondenzen vgl. Hubert Steinke, Der Patron im Netz. Die Rolle des Briefwechsels in wissenschaftlichen Kontroversen, in: Martin Stuber/Stefan Hächler/ Luc Lienhard (Hg.), Hallers Netz. Ein europäischer Gelehrtenbriefwechsel zur Zeit der Aufklärung (Studia Halleriana 9), Basel 2005, S. 441-462, hier S. 443. 
das, was in seinem Auftrag geschah, und sicherte sich zudem die Möglichkeit, jederzeit aus einem Geschäft aussteigen zu können, wenn er mit einem Ergebnis unzufrieden war.

Dass Bessel davon durchaus Gebrauch machte, zeigt ein Beispiel aus der Korrespondenz mit Johann Michael Bockleth, der Bessel 1723 und 1724 auf gleich zwei interessante Münzsammlungen aufmerksam machte, die zum Verkauf standen. Die Kenntnis von diesen Sammlungen hatte Bockleth eher zufällig in Verbindung mit einem anderen Auftrag erlangt, den er für Bessel ausführte und in dem es um die Bestimmung einiger Münzen aus der Göttweiger Sammlung ging.

Bockleth erwies sich für die Klärung von numismatischen Fragen als besonders geeignet, jedoch weniger durch persönliches Expertenwissen, sondern da er sich mit seinem Sitz in Erfurt in unmittelbarer Nähe zu zwei der renommiertesten numismatischen Zentren ihrer Zeit befand, nämlich Arnstadt und Gotha. In Arnstadt hatte Fürst Anton Günther II. von Schwarzburg-Sondershausen (16531716) eine bedeutende Münzsammlung aufgebaut, für deren wissenschaftliche Betreuung Andreas Morell (1646-1703) und als dessen Assistent und Nachfolger Christian Schlegel (1667-1722) gewonnen werden konnten. Diese machten die Sammlung weithin bekannt, sodass Arnstadt auch eine große Dichte an privaten Münzsammlern und Numismatikern aufzuweisen hatte, die im Umkreis der fürstlichen Sammlung forschten und publizierten. Exemplarisch verwiesen sei auf Caspar Sagittarius (1643-1694), Johann Gottfried Olearius (1635-1711) oder Johann Christoph Olearius (1668-1747). Mit dem Verkauf der fürstlichen Münzsammlung an Herzog Friedrich II. von Sachsen-Gotha-Altenburg (1676-1732) im Jahr 1712 und dem damit verbundenen Transfer der Sammlung nach Gotha entstand dort ein weiteres Zentrum für Numismatik, da Schlegel der Sammlung als Kustos erhalten blieb. Sein Nachfolger Christian Sigismund Liebe (1687-1736) sorgte für einen Fortgang der numismatischen Forschung auch am neuen Standort. 34

Unter bislang ungeklärten Umständen hatte Bockleth in Erfurt die Bekanntschaft mit Johann Christian Olearius (1699-1776) gemacht, einem Sohn des Arnstädter Numismatikers und Polyhistors Johann Christoph Olearius. Letzterer wirkte im Brotberuf als Prediger und später Superintendent in Arnstadt, war darüber hinaus aber vor allem als Numismatiker, Historiker und Hymnologe mit zahlreichen Publikationen aktiv. Allein auf dem Gebiet der Numismatik legte er

34 Vgl. Peter Berghaus, Anton Günther II. Graf, seit 1709 Fürst von Schwarzburg-Arnstadt, in: Johann Sebastian Bach und seine Zeit in Arnstadt, hrsg. vom Schlossmuseum Arnstadt, Rudolstadt 2000, S. 137-142; Ders., Das münzsichtige Arnstadt, in: ebd., S. 121-135, hier S. 122 f., 125, 127-129, 132, 134; Ders., Johann Christoph Olearius 17. September 1668 Halle-31. März 1747 Arnstadt, in: Geldgeschichtliche Nachrichten 31 (1996), S. 276-285, hier S. 277 f.; Uta Wallenstein, Das Münzkabinett Gotha im 18. Jahrhundert. Eine prachtvolle fürstliche Sammlung im Spiegel aufklärerischen Denkens, in: Numismatische Zeitschrift 120/121 (2015): Numismatik und Geldgeschichte im Zeitalter der Aufklärung. Beiträge zum Symposium im Residenzschloss Dresden, 4.-9. Mai 2009, hrsg. von Heinz Winter u. a., S. 501-523, hier S. 502, 504. 
bis 1709 siebzehn Schriften vor. ${ }^{35}$ Unklar bleibt, weshalb Olearius seine numismatischen Studien danach einstellte. ${ }^{36}$

Unklar ist auch, zu welchem Zweck sich sein Sohn Johann Christian in Erfurt aufgehalten hatte. Der einzige bisher vorhandene biografische Abriss weist für die Jahre 1720 bis 1724, in welche der Kontakt zu Johann Michael Bockleth fällt, eine Lücke auf. ${ }^{37}$ Und wenngleich der jüngere Olearius in einem Brief als candidat en theologie ${ }^{38}$ bezeichnet wird, ist sein Name in der Matrikel der Universität Erfurt nicht enthalten. ${ }^{39}$ Stattdessen meldet Bockleth im April 1724, der junge Olearius sei damit beauftragt worden, auf ein Vierteljahr das Münzkabinett des Anton Ludwig von Schwarzenfels (1678-1725), Kammerpräsident zu Gotha, zu ordnen und zu verzeichnen. ${ }^{40}$ Diese Anstellung wird durch Olearius selbst bestätigt, wenn dieser darum bittet, an ihn gerichtete Briefe an den Herrn von Schwarzenfels zu adressieren. ${ }^{41}$

Aus den in Göttweig erhaltenen Korrespondenzen - Bockleth leitete auch Briefe aus dem Kontakt mit Olearius an Bessel weiter - zeigt sich, dass Bockleth den jüngeren Olearius gelegentlich mit kleineren Aufträgen versorgte, etwa um von Bessel gewünschte Bücher oder Abschriften von mittelalterlichen Urkunden zu besorgen..$^{42} \mathrm{Am}$ wertvollsten allerdings waren für ihn die Kontakte, die Johann Christian Olearius herstellen konnte, allem voran zu seinem Vater. An diesen trat Bockleth über den Sohn mit der Bitte um eine Expertise zu einigen Münzen aus dem Besitz Bessels heran. Eine Abschrift des Gutachtens hat sich in Göttweig erhalten. ${ }^{43}$ Dem Gutachten ist zu entnehmen, dass Johann Christoph Olearius Abbildungen von drei Münzen erhalten hatte, von denen er eine Heinrich dem Löwen (1129/30-1195) und eine Heinrich I. „dem Vogler“ (ca. 876-936) zuordnen konnte. $\mathrm{Zu}$ der letzten Münze konnte er nicht mehr sagen, als dass er sie für eine gotische Münze des 5. oder 6. Jahrhunderts hielt, die nach dem Vorbild byzantinischer Kaisermünzen dieser Zeit in Italien geschlagen worden war. Eine genauere

35 Eine Auflistung findet sich bei Berghaus, Olearius (wie Anm. 34), S. 280 f. Vgl. auch Wolfgang Tittelbach-Helmrich, Die Oleariuspastoren als Geistliche der Bachkantoren in Arnstadt, in: Bach in Arnstadt (wie Anm. 34), S. 143-163, hier S. $151 \mathrm{f}$.

36 Peter Berghaus vermutet einen Zusammenhang mit dem Verkauf der fürstlichen Münzsammlung; vgl. Berghaus, Olearius (wie Anm. 34), S. 282; Wallenstein, Münzkabinett (wie Anm. 34), S. 502.

37 Vgl. Tittelbach-Helmrich, Oleariuspastoren (wie Anm. 35), S. 157.

38 Anna Margaretha Schlegel an Johann Christian Olearius, 16. Februar 1724: Stiftsbibliothek Göttweig, Cod. 691, fol. 71v.

39 Vgl. Wiegand, Namensverzeichnis (wie Anm. 28), S. 197.

40 Johann Michael Bockleth an Gottfried Bessel, 15. April 1724: Stiftsbibliothek Göttweig, Cod. 691, fol. 194r $-195^{\mathrm{v}}$.

41 Johann Christian Olearius an Johann Michael Bockleth, 19. Juni 1724: ebd., fol. $202^{\mathrm{r}}-203^{\mathrm{v}}$.

42 Johann Michael Bockleth an Gottfried Bessel, 15. April 1724: ebd., fol. 194r ${ }^{\mathrm{r}} 1 \mathrm{5}^{\mathrm{v}}$; Johann Christian Olearius an Johann Michael Bockleth, 19. Juni 1724: ebd., fol. $202^{\mathrm{r}}-203^{\mathrm{v}}$.

43 Ebd., fol. $64^{\mathrm{r}}-66^{\mathrm{v}}$. 
Zuordnung war wegen der fehlenden Umschrift und der schlechten Qualität des Münzstempels nicht möglich. Als Dank für diese und weitere Expertisen, zu denen sich die Gutachten jedoch nicht erhalten haben, ließ Bockleth Johann Christoph Olearius ein Jahr später sechs Flaschen ungarischen Rotweins zukommen. ${ }^{44}$

Einige Monate nach der ersten Kontaktaufnahme für das Gutachten zu den drei Münzen meldete Bockleth an Bessel, er habe ein Schreiben des jüngeren Olearius erhalten, in dem dieser Informationen zum Münzkabinett seines Vaters mitteilte und auch eine Übersicht desselben vorlegte. ${ }^{45}$ Es ist dies die erste Meldung zur privaten Münzsammlung des Johann Christoph Olearius und damit verbunden war offenbar bereits die Möglichkeit eines Verkaufs. Bockleth und der jüngere Olearius erbaten von Gottfried Bessel weitere Anweisungen. Doch dieser zögerte, denn etwa zeitgleich war ihm auch von der Verfügbarkeit der Münzsammlung des 1722 verstorbenen Christian Schlegel berichtet worden. Schlegel war, wie bereits erwähnt, als Kustos der fürstlichen Arnstädter Münzsammlung gemeinsam mit dieser nach Gotha gelangt. Darüber hinaus handelte es sich bei ihm aber auch um einen Studienkollegen von Johann Christoph Olearius, weshalb davon auszugehen ist, dass zwischen den Familien auch ein persönlicher Kontakt bestanden hatte. Es verwundert also nicht, dass der jüngere Olearius den Kontakt zu Schlegels Witwe Anna Margaretha (fl. 1724) herstellen konnte, und auch nicht, dass er die weiteren Schritte nicht an Bockleth abgab, sondern selbst mit ihr verhandelte, nachdem Bessel seine Zustimmung dazu gegeben hatte.

Die erste erhaltene Stellungnahme der Anna Margaretha Schlegel datiert vom 16. Februar 1724, worin sie angibt, immer noch im Besitz des Münzkabinetts ihres verstorbenen Mannes zu sein, das überdies noch vollständig erhalten sei, genauso wie er es ihr hinterlassen habe. Den Umfang der Sammlung beziffert sie mit 1510 Stück sowie etwa 2000 Medaillen, deren Erhaltungszustand insgesamt ausgezeichnet sei, weshalb, so die Witwe, ein jeder Käufer die Sammlung bedenkenlos erwerben könne. ${ }^{46}$ Einen Katalog konnte sie mangels eines geeigneten Bearbeiters nicht bereitstellen, woraus ersichtlich wird, dass bereits Christian Schlegel kein Verzeichnis seiner Münzsammlung angelegt hatte. Jedoch verfügte seine Witwe über einen Katalog der Bücher ihres verstorbenen Mannes, den sie zur Ansicht übermittelte. ${ }^{47}$ Eine Preisvorstellung definierte die Witwe vorerst noch nicht, deutete aber an, in diesem Punkt verhandlungsbereit zu sein.

44 Johann Michael Bockleth an Gottfried Bessel, 14. März 1724: Stiftsbibliothek Göttweig, Cod. 691, fol. $72^{\mathrm{r}}-73^{\mathrm{v}}$.

45 Johann Michael Bockleth an Gottfried Bessel, 23. September 1723: ebd., fol. 83r ${ }^{\mathrm{r}} 84^{\mathrm{v}}$. Der Brief von Olearius an Bockleth hat sich nicht erhalten.

46 Anna Margaretha Schlegel an Johann Christian Olearius, 16. Februar 1724: ebd., fol. $71^{\mathrm{r}-\mathrm{v}}$.

47 Der Katalog hat sich nicht erhalten, wohl weil Anna Margaretha Schlegel ihn, wie auch ein späteres Verzeichnis des Mineralien- und Kuriositätenkabinetts, zurückverlangte. 
Aus dieser ersten Notiz wird deutlich, dass neben der Münzsammlung Christian Schlegels noch weitere Teile seiner Sammlung zum Verkauf standen. Vor allem aber zeigt sich, dass zwei Jahre nach seinem Tod noch kein Käufer gefunden worden war.

Johann Michael Bockleth leitete das Schreiben der Witwe Schlegel an Gottfried Bessel weiter, sah sich jedoch gezwungen, kurz darauf einige der darin gemachten Angaben zu revidieren. Denn es hatte sich herausgestellt, dass das Münzkabinett nicht aus den von Schlegel angegebenen 1510 Stück bestand, sondern etwa 4000 bis 5000 Stück umfasste, was sich negativ auf den Preis auswirken würde. In jedem Fall verlangte die Witwe nun 2000 Reichstaler und war bislang zu keiner Reduktion bereit. Was den fehlenden Katalog der Sammlung betraf, so müsste, nach Meinung Bockleths, ein Käufer dafür mindestens 50 Reichstaler bereitstellen und noch dazu selbst einen geeigneten Bearbeiter finden. Außer Johann Christian Olearius kam seiner Ansicht nach niemand für diese Aufgabe in Frage. Da sich dieser jedoch in gegenwärtig schlechter Verfassung befand, konnte man ihn unmöglich damit beauftragen. Darüber hinaus würde die Erstellung eines Kataloges mit Sicherheit mehrere Jahre in Anspruch nehmen. ${ }^{48}$ Über die Bibliothek Schlegels wusste Bockleth dafür Erfreulicheres zu berichten: sie enthielt einige interessante Stücke, darunter seltene mittelalterliche Handschriften. ${ }^{49}$

Trotz dieses negativen Nachsatzes waren sowohl Bessel als auch die Witwe Schlegel vorerst weiter an einem möglichen Geschäft interessiert, denn nur wenige Wochen nach ihrem ersten Schreiben meldete sie sich erneut bei Johann Christian Olearius..$^{50}$ Dieses Mal übersandte sie einen Katalog des Mineralien- und Kuriositätenkabinetts ihres verstorbenen Mannes, den sie sich aber nach erfolgter Durchsicht zurückerbat, und einen über die Bücher, der auch eine Preisliste enthielt. Was die Münzen betraf, so hatte sie die Sammlung noch einmal in Augenschein genommen und festgestellt, dass es sich um 5730 Stück unterschiedlichster Sorten handelte. Was ihre Preisvorstellung betraf, so verlangte sie für die Münzen weiterhin 2000 Reichstaler, für die Kuriositäten 300 Reichstaler und für die Mineralien 60 Reichstaler.

Im Gegensatz zum eher nüchtern gehaltenen ersten Schreiben warb Anna Margaretha Schlegel nun eindeutig um Bessel als Käufer und versuchte unterschwellig, ihn zu einem Kauf zu bewegen, indem sie von anderen Interessenten berichtete, welche die Sammlung in Augenschein genommen hätten. Zuletzt verlieh sie ihrer Hoffnung Ausdruck, die gesamte Sammlung samt Bibliothek geschlossen an einen Käufer abgeben zu können. $\mathrm{Zu}$ diesem Zweck wäre sie sogar bereit, Abstriche beim Preis zu machen.

48 Johann Michael Bockleth an Gottfried Bessel, 11. März 1724: Stiftsbibliothek Göttweig, Cod. 691, fol. 76 $6^{\mathrm{r}}-77^{\mathrm{v}}$.

49 Johann Michael Bockleth an Gottfried Bessel, 14. März 1724: ebd., fol. $72^{\mathrm{r}}-73^{\mathrm{v}}$.

50 Anna Margaretha Schlegel an Johann Christian Olearius, 16. März 1724: ebd., fol. $190^{\mathrm{r}}-191^{\mathrm{v}}$. 
Johann Michael Bockleth kam nicht umhin, auch dieses Mal die Angaben der Witwe Schlegel zu überprüfen und zu korrigieren. Seiner Einschätzung ihres übersandten Katalogs zum Mineralien- und Kuriositätenkabinett nach war dies kaum mehr als 120 Reichstaler wert, die Bücher schätzte er auf insgesamt 80 Reichstaler, würde ihr aber vorerst nur 60 Reichstaler dafür bieten, um zu sehen, ob sie tatsächlich zu Preisverhandlungen bereits war. Unterdessen hielt er Kontakt $\mathrm{zu}$ Johann Christoph Olearius für weitere Gutachten von Münzen. ${ }^{51}$

In weiterer Folge kam der Kontakt zu Anna Margaretha Schlegel vorläufig zum Erliegen. Wie aus der weiteren Korrespondenz Bockleths mit Bessel ersichtlich ist, hatte Bockleth ihr im Auftrag Bessels für die Bücher die erwähnten 60 Reichstaler geboten und für die Münzen 1000 Reichstaler. Für die Mineralien und Kuriositäten wurde gar kein Gebot abgegeben. Da die Witwe auf diese Preisvorschläge nicht reagierte, versprach Johann Christian Olearius, sie persönlich aufzusuchen. Er hielt sich mittlerweile ebenfalls in Gotha auf, um das Münzkabinett des Anton Ludwig von Schwarzenfels zu ordnen. Wie Bockleth berichtete, plante Olearius zudem für das kommende Jahr eine Reise nach Wien, was Bockleth dazu veranlasste, Bessel den Vorschlag zu unterbreiten, ihn bei dieser Gelegenheit nach Göttweig einzuladen, um sich mit ihm über das dortige Münzkabinett auszutauschen. ${ }^{52}$

Was die Sammlung Schlegel betraf, so zeitigte auch der persönliche Einsatz von Johann Christian Olearius keinen Erfolg. Trotz aller Überredungsversuche weigerte sich die Witwe, das Münzkabinett ihres verstorbenen Mannes um die gebotenen 1000 Reichstaler zu verkaufen. Stattdessen forderte sie sämtliche bisher übersandten Kataloge zurück, sodass nach vier Monaten der Verhandlungen diese erfolglos eingestellt werden mussten. ${ }^{53}$ Dass auch andere Interessenten kein Glück hatten, zeigt eine Bemerkung Bockleths, wonach auch das Gebot des Bibliothekars Johann Friedrich Schannat (1683-1739), der immerhin 1800 Reichstaler für die Münzsammlung geboten hatte, von Anna Margaretha Schlegel abgelehnt worden war. ${ }^{54}$

51 Johann Michael Bockleth an Gottfried Bessel, 23. März 1724: Stiftsbibliothek Göttweig, Cod. 691, fol. 188r $-189^{v}$.

52 Johann Michael Bockleth an Gottfried Bessel, 15. April 1724: ebd., fol. 194r $-195^{\mathrm{v}}$.

53 Johann Christian Schlegel an Johann Michael Bockleth, 19. Juni 1724: ebd., fol. $202^{\mathrm{r}}-203^{\mathrm{v}}$.

54 Johann Michael Bockleth an Gottfried Bessel, 29. Juli 1724: ebd., fol. 196 ${ }^{\mathrm{r}}-197^{\mathrm{v}}$. Der Luxemburger Johann Friedrich Schannat war seit 1721 Bibliothekar und Hofhistoriograf des Hochstiftes Fulda, wo er im Auftrag des Fürstabts Konstantin von Buttlar (16791726) die Geschichte des Hochstiftes aufarbeitete. Seine Recherchen für diese 1729 erschienene „Historia Fuldensis“ führten ihn auch nach Gotha und Erfurt; vgl. WilHELM ENGEL, Johann Friedrich Schannat (1683-1739). Leben - Werk - Nachlaß, I. Teil: Leben und Werk, in: Archivalische Zeitschrift 44 (1936), S. 24-103, hier S. 46-59; Walter Goztze, Aus der Frühzeit der methodischen Erforschung deutscher Geschichtsquellen. Johann Friedrich Schannat und seine Vindemiae Literariae (Berliner Studien zur neueren Geschichte 7), Würzburg 1939, S. 7 f.; JoËLle WeIs, Johann Friedrich Schannats „Historia Fuldensis“ und der Gelehrtenstreit mit Johann Georg von Eckhart, 
Das Scheitern der Bemühungen um die Münzsammlung Schlegels lenkte Bessels Interesse nun aber zurück zu jener von Johann Christoph Olearius, die ihm Monate zuvor bereits unverbindlich angeboten worden war. Wie Bockleth Bessel versicherte, bestand das Angebot nach wie vor und Johann Christian Olearius hatte sich erboten, detailliertere Informationen von seinem Vater einzuholen und bereitzustellen. Wenngleich es an Bessel war, seine Zustimmung zum Start ernsthafter Verhandlungen zu geben, so kam Bockleth nicht umhin zu bemerken, dass er die Sammlung Schlegels zwar für reichhaltiger an Brakteaten und Solidi hielt, jene von Olearius aber insgesamt für wertvoller. ${ }^{55}$ Ohne damit offen eine Empfehlung ausgesprochen zu haben, hatte Bockleth seine Meinung zum weiteren Vorgehen mitgeteilt.

Gottfried Bessel teilte diese Ansicht und gab entsprechende Anweisungen und schon am 26. August 1724 wurde ihm eine erste Aufstellung des Münzkabinetts übermittelt, in der Vater und Sohn Olearius in 27 Punkten die einzelnen Untergruppen der Sammlung zusammenfassten. ${ }^{56}$ Diese beinhaltete demnach 1. antike römische Münzen von Julius Cäsar (100 v. Chr.-44 v. Chr.) bis auf Kaiser Honorius (384-423), 2. Münzen deutscher Könige und Kaiser von Karl dem Großen (747/48-814) bis auf Ludwig IV. (1281/82-1347), 3. etwa 200 Brakteaten, 4. Solidi von Bischöfen und Erzbischöfen, 5. einige antike gotische Münzen, 6. Münzen mit Abbildungen von Heiligen, 7. neuzeitliche Kaisermünzen, 8. Papstmünzen, 9. Münzen der Königreiche Portugal, Spanien, Frankreich, Ungarn, Schweden und Böhmen, 10. Münzen aus Russland, Persien, Türkei, Indien, Algerien und anderen orientalischen Gebieten, 11. Münzen italienischer Herzogtümer, 12. Münzen aus Holland, 13. Münzen der unterschiedlichen sächsischen Linien ab Friedrich I. (1257-1323), 14. Münzen der Herrschaft Brandenburg-Preußen, 15. Münzen der Herrschaft Braunschweig-Lüneburg, 16. Münzen der Herrschaft Schwarzburg, 17. Klippen, 18. Münzen anderer deutscher Herrschaften, darunter Mansfeld, Stollberg und Heimberg, 19. Münzen von Herzogtümern außerhalb des Reichs, 20. Varia, darunter hebräische Münzen, 21. Münzen von Städten, 22. Medaillen berühmter Männer, 23. antike römische Kupfermünzen, 24. unterschiedliche neuere Kupfermünzen, 25. Zinnabgüsse von Nürnberger Pfennigen und Medaillen, 26. Jetons aus Kupfer und Messing, 27. Bleiabgüsse. Das Gesamtgewicht der Sammlung wurde mit 550 Lot bemessen, allerdings ohne Angabe, welches Lot für die Berechnung herangezogen wurde. Eine Preisvorstellung enthielt diese erste Aufstellung nicht, doch Bockleth ließ in seinen die Auflistung begleitenden Zeilen wissen, dass Johann Christian Olearius seinen Vater dazu hatte bringen können,

in: Cornelia Faustmann/Gottfried Glaßner/Thomas Wallnig (Hg.), Melk in der barocken Gelehrtenrepublik. Die Brüder Bernhard und Hieronymus Pez, ihre Forschungen und Netzwerke (Thesaurus Mellicensis 2), Melk 2014, S. 197-204, hier S. $198 \mathrm{f}$.

55 Johann Michael Bockleth an Gottfried Bessel, 29. Juli 1724: Stiftsbibliothek Göttweig, Cod. 691, fol. 196r $-197 \mathrm{v}$.

56 Ebd., fol. 87r-v. 
die Münzsammlung um 1200 Reichstaler anzubieten. ${ }^{57}$ Es dürfte kein Zufall sein, dass diese Summe im Bereich dessen lag, was Gottfried Bessel für die Sammlung Schlegel zu zahlen bereit gewesen wäre.

Bereits einen Monat später lieferten Vater und Sohn Olearius eine weitere, nun weitaus detailliertere Aufstellung des Münzkabinetts, um die sie offenbar gebeten worden waren. Johann Christian Olearius schien es wichtig, dabei zu betonen, dass die Sammlung mit Ausnahme einiger weniger Abgüsse, die jedoch optisch klar als solche erkennbar waren, ausschließlich aus Originalen bestand. Außerdem hatte sein Vater eingewilligt, gemeinsam mit den Münzen auch die von Bessel angefragte numismatische Fachliteratur zu verkaufen. Als Rechtfertigung für diesen Schritt fügte Johann Christian Olearius an, dass sein Vater die betreffenden Titel auch in der fürstlichen Bibliothek benutzen konnte und deshalb in seiner Privatbibliothek entbehren konnte. ${ }^{58}$

Allem Anschein nach hatte Johann Christoph Olearius nicht nur seine numismatischen Studien eingestellt, sondern darüber hinaus auch das Interesse an diesem Fach verloren, was Peter Berghaus' These von einem Zusammenhang mit dem Verkauf der Münzsammlung Anton Günthers II. stützt. ${ }^{99} \mathrm{Da}$ sich in der Korrespondenz Bockleths mit Bessel aber auch Hinweise finden, wonach Olearius einem Verkauf seiner Antiquitäten und Naturalien nicht abgeneigt war, müssen auch andere Gründe, etwa finanzieller Natur, in Erwägung gezogen werden. Wenngleich ein Großteil der Sammlung des Johann Christoph Olearius in seinem Besitz verblieb und nach seinem Tod unter den Erben aufgeteilt wurde, kann und darf daraus nicht notwendigerweise geschlossen werden, dass diese Objekte nicht bei günstiger Gelegenheit den Besitzer hätten wechseln können. In den mit Johann Michael Bockleth geführten Verhandlungen ging es jedoch ausschließlich um die Münzsammlung, was ein wenig verwundert, da Gottfried Bessel durchaus auch an mathematischen Instrumenten, Kunstwerken und Naturalien interessiert war und seinem Schwager entsprechende Kaufaufträge erteilte. Weshalb er an den restlichen Stücken der Sammlung Olearius nicht interessiert war, bleibt offen. Unklar bleibt auch, ob im Fall der Münzen tatsächlich die gesamte Münzsammlung zum Verkauf gelangte oder nur ein Teil davon. Denn wie aus der zweiten Aufstellung zur Sammlung hervorgeht, wurden Bessel zwar Silber- und Kupfermünzen sowie Zinn- und Bleiabgüsse angeboten, jedoch keine Goldmünzen. Dass sich keine solchen in der Sammlung befunden hätten, erscheint unwahrscheinlich. ${ }^{60}$ Doch

57 Johann Michael Bockleth an Gottfried Bessel, 26. August 1724: ebd., fol. 204r ${ }^{\mathrm{r}}-205^{\mathrm{v}}$.

58 Johann Christian Olearius an Johann Michael Bockleth, 21. September 1724: ebd., fol. $85^{\mathrm{r}}-86^{\mathrm{v}}$.

59 Vgl. Anm. 36.

60 Nach Berghaus, Olearius (wie Anm. 34), S. 283 f. soll der Dresdner Numismatiker Christian Jacob Götz (1756-1837) von einem Privatmann einige Goldmünzen aus dem Besitz von Johann Christoph Olearius erworben haben. Durch den Verkauf der Sammlung Götz an Rudolf Benno Römer (1802-1871) sollen die Münzen in weiterer Folge an das Münzkabinett der heute Staatlichen Kunstsammlungen Dresden gelangt sein. Nachprüfbare Belege finden sich bei Berghaus nicht. 
auch ohne Goldmünzen war das Konvolut, das Bessel zum Kauf angeboten wurde, beeindruckend. Insgesamt bestand es aus 3087 Einzelstücken, von denen 2251 aus Silber, 461 aus Kupfer, 292 aus Zinn und 83 aus Blei waren. ${ }^{61}$

Im Gegensatz zur ersten an Bessel versandten Auflistung, die grob die Untergruppen der Sammlung benannte, war die zweite insofern detailreicher, als dass sie Angaben zu Metallen und Stückzahlen enthielt. Auch wurden die 27 Gruppen der ersten Aufstellung noch genauer geschieden, sodass die zweite Aufstellung insgesamt 53 Positionen umfasste. Wie Johann Christian Olearius zugesagt hatte, waren die Abgüsse explizit ausgewiesen worden. Allerdings war ihm und seinem Vater auch ein Fehler unterlaufen, den Johann Christoph Olearius wenige Tage später korrigieren musste. Denn die noch in der ersten Aufstellung unter Punkt 8 genannten Papstmünzen waren in der zweiten Aufstellung nicht mehr enthalten gewesen. Da diese aber zwischenzeitlich aufgefunden worden waren, konnte er angeben, dass es sich um 14 Silber-, 5 Kupfer- und 22 Bleimünzen, also insgesamt 41 Stück handelte. Darüber hinaus konnte sich Olearius den Seitenhieb nicht verkneifen, dass seine Münzsammlung nun nachweislich vielseitiger war als jene von Christian Schlegel.62

Dieser Nachtrag ist in mehrfacher Hinsicht interessant. Zum einen lässt sich ein klein wenig über die Aufbewahrung der Münzen sagen, denn offenbar waren nicht alle Münzen im selben Behältnis gelagert worden. Zum anderen wird deutlich, dass Johann Christoph Olearius (und sein Sohn) erst für die zweite Aufstellung direkt mit den Münzen gearbeitet hatten, wohingegen die erste Aufstellung möglicherweise auf Basis älterer Notizen oder gar frei aus dem Gedächtnis des Besitzers entstanden sein dürfte. Auch wird eine gewisse Konkurrenzsituation zwischen Johann Christoph Olearius und Christian Schlegel angedeutet, wobei unklar bleibt, ob eine solche auch zu Lebzeiten der beiden ehemaligen Studienkollegen bestanden hatte, oder an dieser Stelle rein auf die Münzsammlungen bezogen $\mathrm{zu}$ verstehen ist, eventuell vielleicht auch mit einer kleinen Spitze gegen Gottfried Bessel versehen, der von Olearius zu Schlegel wechselte, um dann wieder zu Olearius zurückzukehren. Zuletzt lässt sich auch etwas über die persönliche Situation der Familie Olearius sagen, denn in einem Nachsatz erläutert Johann Christoph Olearius, weshalb nicht wie gewohnt sein Sohn die Korrespondenz übernehmen konnte: Johann Christian Olearius stand im Begriff, zum Prediger in Arnstadt bestellt zu werden, und hielt sich zu diesem Zweck in Gotha auf.63

Dass Gottfried Bessel sich an diesem Punkt bereits zu einem Kauf der Münzsammlung Olearius entschlossen hatte, steht außer Zweifel, dennoch wollte er sich dem Verkäufer gegenüber absichern und die Ware vor Vertragsabschluss in Augenschein nehmen. Da dies aufgrund der räumlichen Distanz für ihn persön-

61 Stiftsbibliothek Göttweig, Cod. 691, fol. 110r-111v.

62 Johann Christoph Olearius an Johann Michael Bockleth, 25. September 1724: ebd., fol. $104^{\mathrm{r}}-105^{\mathrm{v}}$.

63 Dieser Karriereschritt ist auch in der Biografie von Johann Christian Olearius belegt; vgl. Tittelbach-Helmrich, Oleariuspastoren (wie Anm. 35), S. 157. 
lich nicht möglich war, wurde Johann Michael Bockleth mit einer Inspektion der Sammlung beauftragt, was diesen vor gewisse Schwierigkeiten stellte, da er kaum über numismatische Kenntnisse verfügte. Doch Bockleth wusste sich zu helfen und stattete sich mit dem Werk des Hubert Goltzius (1526-1583) aus, das er mit nach Arnstadt zu Johann Christoph Olearius nahm. Mit diesem wollte er, wie er vorab berichtete, vor allem die römischen Kaisermünzen überprüfen. ${ }^{64}$

Ein Gutachten Bockleths zur Münzsammlung Olearius hat sich nicht erhalten und wurde höchstwahrscheinlich von ihm auch nicht erstellt. Vielmehr ist davon auszugehen, dass seine Rolle primär einen Abgleich der Sammlung mit der zweiten Auflistung hinsichtlich der angegebenen Stückzahlen und Münzmetalle vorsah sowie eventuell eine Beurteilung des allgemeinen Erhaltungszustandes. Weshalb er so großes Gewicht auf die Beurteilung der römischen Kaisermünzen legte, bleibt unklar. Womöglich war Bessel die Beschreibung dieses Postens in den beiden Auflistungen Olearius' zu ungenau und er verlangte nach detaillierteren Angaben.

Das Ergebnis von Bockleths Inspektion schien alle Parteien zufriedenzustellen, denn schon am 23. Oktober 1724 wurde der Kaufvertrag zwischen Johann Christoph Olearius und Gottfried Bessel aufgesetzt. Darin erklärte Olearius, sein Münzkabinett vorbehaltlich der Zahlung des vereinbarten Kaufpreises von 800 Reichstalern Gottfried Bessel zu übergeben, überlaßen und zu verkauffen mit allem, was diesem bisher davon gezeigt worden war. ${ }^{65}$ Das Kabinett bestand aus zwei Kästen und zusätzlich einigen Brettern mit abgüßen, geprägen und dergleichen und sollte vorerst nach Erfurt geliefert werden. Im Frühjahr oder Sommer des kommenden Jahres sollte dann der jüngere Olearius die Sammlung anlässlich einer geplanten Reise nach Österreich nach Göttweig begleiten und bei dieser Gelegenheit einen Katalog anfertigen. Für die zusätzlichen Reisekosten war er vom Käufer zu entschädigen. Die beiden besagten Münzkästen waren bereits auf Kosten des Käufers für 30 Louis d'Or angefertigt worden, was Olearius ebenfalls im Vertrag festhielt. Ebenfalls festgehalten wurde, dass der Vertrag in duplo ausgestellt wurde, sodass jeder Vertragspartner über ein Exemplar verfügte, das von beiden unterschrieben und besiegelt wurde. In Göttweig hat sich deshalb nur der von Olearius unterfertigte Vertrag mit dessen Unterschrift und Siegel erhalten. Bessel sah es offenbar nicht für notwendig an, das bei ihm verbleibende Exemplar, wie von Olearius gewünscht, selbst zu unterschreiben und zu siegeln, weshalb seine Unterfertigung auf dem Dokument fehlt.

Schon einen Tag nach Vertragsschluss meldete Bockleth, die 30 Reichstaler Anzahlung geleistet zu haben und in einigen Tagen die restlichen 770 Reichstaler

64 Johann Michael Bockleth an Gottfried Bessel, 10. Oktober 1724: Stiftsbibliothek Göttweig, Cod. 691, 101 ${ }^{\mathrm{r}}-102^{\mathrm{v}}$. Bei dem Werk von Goltzius handelt es sich mit großer Wahrscheinlichkeit um Hubert Goltzius, De re nummaria antiqua, opera quae extant universa, 5 Bde., Antwerpen 1708. Die Münzen der römischen Kaiserzeit wurden in Band 2 behandelt; vgl. Christian E. Dekesel/Yvette M. M. Dekesel-De Ruyck, A bibliography of 18 th century numismatic books, Bd. 3, London 2013, S. 33-38.

Stiftsbibliothek Göttweig, Cod. 691, fol. 126r ${ }^{\mathrm{r}}-127^{\mathrm{v}}$. 
überbringen und das Münzkabinett zu sich liefern zu lassen.66 Da er sich die Summe aber leihen musste, bat er Gottfried Bessel um baldige Refundierung. Nun klärt sich auch, weshalb Bockleth im Vorfeld so großes Augenmerk auf die römischen Kaisermünzen in der Sammlung Olearius gelegt hatte: diese bildeten, so Bockleth, im Kabinett von Olearius nämlich keine eigene Serie, sondern waren mit anderen Münzen vermengt. Leider würden auch einige Kaiser fehlen, jedoch versprach Bockleth, sein Möglichstes zu tun, um diese anderweitig aufzutreiben. Auch hätte er Johann Christian Olearius 100 Reichstaler versprochen, wenn er im fürstlichen Münzkabinett zu Gotha, das unter anderem über eine komplette Serie der römischen Kaiser verfügte, Münzen fände, die Bessel bisher unbekannt waren. Würde Bessel ihm eine größere Summe zur Verfügung stellen, könnte er überdies noch mehr Münzen für diesen einkaufen. Doch davon sah Bessel ab.

Interessant erscheint in diesem Zusammenhang die Notiz, Bockleth habe Johann Christoph Olearius signalisiert, dass Bessel eventuell auch an dessen Naturalienkabinett interessiert sein könnte. Er hatte in dieser Sache jedoch vorerst keine weiteren Schritte unternommen, aus Angst, Olearius hätte vom Verkauf der Münzen Abstand genommen, falls kein Handel über die Naturalien zustande gekommen wäre. Weiters meldete Bockleth auch die Möglichkeit zum Erwerb einiger Antiquitäten aus dem Besitz Olearius'.67

Doch von Verhandlungen über weitere Ankäufe von Objekten aus dem Besitz von Olearius findet sich in der Korrespondenz zwischen Bockleth und Bessel tatsächlich keine Spur mehr, weshalb davon auszugehen ist, dass Bessel weitere Verhandlungen in dieser Sache nicht autorisierte. Stattdessen sandte er die Kaufsumme für die Münzen per Wechsel nach Erfurt, deren Erhalt durch Bockleth Johann Christoph Olearius am 27. Oktober 1724 quittierte. ${ }^{68}$ Schon am nächsten Tag wurde das Münzkabinett nach Erfurt geliefert. ${ }^{69}$

Obwohl seitens Johann Christoph Olearius' die (letztlich nicht erfüllte) Hoffnung bestanden haben mag, noch weitere Teile seiner Privatsammlung an Gottfried Bessel zu verkaufen, blieb er diesem zumindest als Gutachter erhalten, so etwa für eine Sammlung von Brakteaten, die Bockleth für Bessel erworben hatte. ${ }^{70}$ $\mathrm{Zu}$ seinem Sohn Johann Christian Olearius, dessen geplanter Reise nach Göttweig und dem zu erstellenden Katalog zur Münzsammlung des Vaters gibt es keinen weiteren Hinweis. Die Korrespondenz Bockleths mit Bessel schweigt dazu ebenso wie zeitgenössische Göttweiger Quellen, in denen der Aufenthalt eines Gastes

66 Johann Michael Bockleth an Gottfried Bessel, 24. Oktober 1724: Stiftsbibliothek Göttweig, Cod. 691, fol. 98 r-99v.

67 Johann Michael Bockleth an Gottfried Bessel, 28. Oktober 1724: ebd., fol. 100r-v.

68 Ebd., fol. $127^{r-v}$.

69 Vgl. Anm. 67.

70 Johann Michael Bockleth an Gottfried Bessel, 4. April 1725: Stiftsbibliothek Göttweig, Cod. 691, fol. 118 ${ }^{\text {r-v}}$; Johann Michael Bockleth an Gottfried Bessel, 25. September 1725: ebd., fol. 114r-v; Johann Michael Bockleth an Gottfried Bessel, 13. Oktober 1725: ebd., fol. $112^{\mathrm{r}}-113^{\mathrm{v}}$. 
vermerkt worden wäre, etwa das Tagebuch des Priors Gregor Schenggl (16841750). ${ }^{71}$

Im Lichte des weiteren Lebenswegs von Johann Christian Olearius wird rasch ersichtlich, weshalb er die ihm zugedachte Aufgabe nicht mehr erfüllen konnte: wie bereits erwähnt, wurde Johann Christian Olearius zum Prediger an der Neuen Kirche in Arnstadt (heute: Bachkirche) bestellt. Dieses Amt übte er bis zum Jahr 1736 aus, ehe er zum Subdiakon und Bibliothekar der Kirchenbibliothek zu Arnstadt ernannt wurde. Nach dem Tod seines Vaters im Jahr 1747 folgte er diesem als Diakon nach und wurde 1755 schließlich zum Archidiakon bestellt. ${ }^{72}$ Damit war Johann Christian Olearius, der in den Jahren vor diesem Karriereschub vor allem mit kleineren Rechercheaufträgen für Johann Michael Bockleth und einer temporären Anstellung bei Anton Ludwig von Schwarzenfels sein Auskommen hatte finden müssen, nicht nur langfristig versorgt, sondern auch mit Aufgaben betraut worden, die seine ständige Präsenz in Arnstadt erforderten. Eine Reise in ein österreichisches Stift für kurzfristige Katalogisierungsarbeiten war für ihn deshalb weder organisatorisch möglich noch finanziell erforderlich.

Stattdessen fertigte Johann Christian Olearius in seiner Funktion als Bibliothekar der Arnstädter Kirchenbibliothek zwei umfangreiche Kataloge der dortigen Bestände an. ${ }^{73}$ Vor allem der spätere Katalog aus dem Jahr 1771 ist im Hinblick auf die Privatsammlung von Johann Christoph Olearius eine interessante Quelle, da er über deren weiteren Verbleib Aufschluss gibt. So vermachte Johann Christoph Olearius der Kirchenbibliothek testamentarisch seine Sammlung an Schriften Martin Luthers (1483-1546), die ausschließlich aus Ausgaben bestand, die noch zu Lebzeiten des Reformators erschienen waren. Die übrige Sammlung hinterließ er seinen Erben, und zwar seiner Ehefrau und seinen Kindern. Im Fall von bereits verstorbenen Kindern rückten deren Erben nach. ${ }^{74}$

Noch aufschlussreicher als der Katalog der Arnstädter Kirchenbibliothek ist ein von Johann Gottfried Müller (1729-1792), einem Enkel des Johann Christoph Olearius, verfasstes Verzeichnis über die Sammlung des Großvaters, das drei Jahre

71 Gregor Schenggl, Diarium Monasterii Gottwicensis, Bd. 1-5, Göttweig 1718-1748: Stiftsarchiv Göttweig Cod. Ser. Nov., S. 90-94; vgl. auch Lechner, Göttweig (wie Anm. 2), S. 832.

72 Vgl. Tittelbach-Helmrich, Oleariuspastoren (wie Anm. 35), S. 157.

73 Johann Christian Olearius, Kurtze doch hinlängliche Nachricht von der öffentlichen Kirchen-Bibliothek in Arnstadt, derselben Stiftung, Fortsetzung und Erneuerung, auch ihren gegenwärtigen Zustand betreffend, welche nebst dem Verzeichnis der Bücher, so darinne befindlich, in nachstehenden bekannt machet, Arnstadt 1746; Ders., Erste Fortsetzung des Verzeichnisses derjenigen Bücher, welche in der öffentlichen Kirchen-Bibliothek zu Arnstadt befindlich sind, und von 1746 bis 1771 theils gekauft, theils geschenkt worden, Arnstadt 1771. Vom späteren Katalog scheint sich nur noch ein Exemplar in Arnstadt erhalten zu haben: Kirchenbibliothek zu Arnstadt 2129; vgl. Tittelbach-Helmrich, Oleariuspastoren (wie Anm. 35), S. 163.

74 Vgl. Olearius, Erste Fortsetzung (wie Anm. 73), S. 32; Tittelbach-Helmrich, Oleariuspastoren (wie Anm. 35), S. 155 f. 
nach dessen Tod veröffentlicht wurde. ${ }^{75}$ Dabei handelt es sich um keinen Katalog der Sammlung, wie sie zu Lebzeiten ihres Besitzers eingerichtet und aufgestellt worden war, sondern um eine Rekonstruktion derselben auf Basis der Objekte, die sich noch im Besitz der Erben befanden. Die aktuellen Besitzer der Sammlung werden auf der letzten Seite des Drucks aufgeführt. Es handelt sich, nach Müller, um die Witwe, Johann Christoph Olearius' zweite Ehefrau Eleonore Sabine (fl. 1715-1750), den Sohn Johann Christian sowie die Schwiegersöhne Samuel Kaspar Schuhmann († 1754), Johann Friedrich Müller (1699-1752) und die Kinder des bereits verstorbenen Schwiegersohns Georg Volkmar Hartmann (fl. 1729-vor 1750). Bei der Aufzählung der Besitzer verursachte Johann Gottfried Müller gleich mehrere Missverständnisse: so unterlief ihm der Lapsus, seinen Onkel Georg Volkmar Hartmann irrig als Volkmar Heinrich anzugeben. Weit schwerer wiegt jedoch der Eindruck, Johann Christoph Olearius habe seine Sammlung neben seiner aktuellen Ehefrau und dem letzten noch lebenden Sohn seinen Schwiegersöhnen und nicht seinen Töchtern vermacht. Aus der Erwähnung bei Johann Christian Olearius ${ }^{76}$ geht deutlich hervor, dass seine Schwestern gleichberechtigt erbten. Allerdings waren sowohl Juliana Elisabeth († vor 1750), die Ehefrau von Johann Friedrich Müller, als auch Augusta Johanna ( $†$ 1739), die Ehefrau von Georg Volkmar Hartmann, bereits verstorben. Wann Johanna Sophia, die Ehefrau von Samuel Kaspar Schuhmann, verstarb, ist unklar. ${ }^{77}$ Es muss also in Betracht gezogen werden, dass zum Zeitpunkt der Veröffentlichung von Müllers Verzeichnis sämtliche Töchter, die 1747 noch als Erben bedacht worden waren, bereits verstorben waren und die geerbte Sammlung deshalb auf deren Ehemänner übergegangen war.

Zwar zollt Müller diesem Umstand insofern Rechnung, als er sämtliche aktuelle Besitzer in seinem Druck tatsächlich auch als solche bezeichnet und nicht etwa als Erben des Johann Christoph Olearius. Und auch der Hinweis, dass sich die Sammlung 1750 mehrheitlich im Besitz der Schwiegersöhne befand, legt nahe, dass diese über den Umweg ihrer Ehefrauen an die Sammlungen gelangt waren. Ohne den Hinweis im Katalog von Johann Christian Olearius könnte jedoch der Eindruck entstehen, Johann Christoph Olearius habe von vorneherein nicht seine Töchter, sondern an ihrer statt deren Ehemänner bedacht.

Neben den leicht missverständlichen Besitzverhältnissen unterlässt es Müller zudem mehrheitlich, darauf hinzuweisen, welche Sammlungsteile oder Objekte

75 Johann GotTfried Müller, Verzeichnis der vornehmsten Stücke welche in dem nunmehr zertheilten Curiositäten- und Naturalien-Cabinet Johann Christoph Olearii [...] befindlich gewesen sind, Jena 1750.

76 Vgl. Olearius, Erste Fortsetzung (wie Anm. 73), S. 32.

77 Spärliche Angaben zu den Lebensdaten der Töchter finden sich in: Olearius (Johann Christoph), in: Zedler's Universallexikon, Bd. 25, Halle/Leipzig 1740, Sp. 1176-1185, hier Sp. 1179 f. Die jüngste Tochter Johanna Sophia wird darin noch als unverheiratet angegeben, was darauf schließen lässt, dass sie zwischen 1740, dem Erscheinungsjahr des Zedler-Bandes, und 1750, dem Erscheinungsjahr des Verzeichnisses von Müller, heiratete. 
sich im Besitz der einzelnen Erben beziehungsweise deren Familien befanden. Nur fallweise wies er einzelne Objektgruppen einer Familie zu und markierte dabei vor allem jene, die sich im Besitz seiner eigenen befanden.

Insgesamt unterschied Müller 15 Kategorien, wobei er die Objekte der ersten zehn Kategorien unter einem gemeinsamen Oberbegriff zusammenfasste, die verbleibenden fünf jedoch als lose Aneinanderreihung von Objekten ähnlicher Thematik präsentierte, wobei anzumerken ist, dass viele Objekte dieser Kategorien auch den anderen zehn hätten zugeordnet werden können. Ob Müller mit dieser Einteilung eine frühere Ordnung der Sammlung abzubilden suchte oder die Objekte nach eigenem Gutdünken zusammenstellte, ist unklar, zumal er es verabsäumte, seinem Verzeichnis begleitende Worte zur Genese der Sammlung, ihrer Systematik oder ihrer Aufteilung nach dem Tod von Johann Christoph Olearius beizufügen. Auch zu seiner eigenen Arbeitsweise schweigt er, sodass sein Verzeichnis direkt mit der ersten Objektgruppe beginnt.

Die Objekte der ersten zehn Kategorien unterteilte Müller in die Bereiche 1) Sacra, 2) Humana, 3) Varia vestitamenta, vasa, utensilia etc., 4) Arma vetera et recentiora, 5) Animalia, 6) Volatilia, 7) Marina, 8) Insecta, 9) Vegetabilia und 10) Lapides figurati, mineralia, gemmae. Die Rubrik Sacra umfasste dabei Ablassbriefe, Weihebilder, Oblaten mit sakralen Motiven, Urnen und verschiedene Rosenkränze ebenso wie Opfergegenstände und Grabbeigaben heidnischer Kulte. Unter Humana fasste Müller Teile ägyptischer Mumien, missgebildete Föten, verarbeitete menschliche Haut und Skelettteile (vornehmlich Schädel) zusammen. Varia vestitamenta, vasa, utensilia umfasste Kopfbedeckungen, Schuhe, Taschen, Hausrat, Schüsseln, Besteck und Gefäße fremder Kulturen. Die Sektion Arma enthielt Pfeile, Bogen, Speere, Armbrüste, Messer, Schwerter und Teile von Rüstungen. Unter Animalia subsummierten sich Hörner, Geweihe, Tierzähne und Bezoare. Unter Volatilia waren es exotische Vögel, Eier, Schnäbel und Klauen. Unter Marina versammelte Müller Krebse, Schildkröten, Seesterne, Meerespflanzen, Korallen, Schnecken und Muscheln sowie Teile von Delfinen, Walen und Schwertfischen. Die Rubrik Insecta beinhaltete Spinnen, Skorpione, Kröten und Schlangen (entweder im Ganzen oder Teile davon). Vegetabilia versammelte exotische Hölzer und deren Samen, etliche Varianten von Palmzweigen und Zuckerrohr sowie exotische Pflanzen und Gewürze wie Zimt oder Kokosnüsse. Unter lapides figurati, mineralia, gemmae subsummierten sich unterschiedliche Erden, Steinsorten, Steine mit besonderen Farben oder Mustern, Fossilien, versteinerte Samen, Muschelsteine, Ammoniten, Halbedelsteine sowie unterschiedliche Metallproben.

Als elfte Kategorie präsentierte Müller Schwefelprodukte und Alaune, als zwölfte Kategorie versteinertes Getreide und altes Brot und Mehl. Einige bundert alte und neue Kupferstiche bildeten die dreizehnte Kategorie, ${ }^{78}$ Bildnisse von Gelehrten, exotische Schriftproben, Tintenarten und Papiersorten die vierzehnte 
Kategorie und in der letzten Kategorie versammelte Müller optische Geräte, Kunstwerke und ein Brettspiel, auf dem Kaiser Karl V. (1500-1558) mehrmals gespielt haben soll.

Die nicht kongruente Struktur wird anhand der letzten fünf Kategorien besonders deutlich: so hätten die Objekte der Kategorie elf problemlos mit jenen der Kategorie zehn vereint werden können, und auch das versteinerte Getreide aus Kategorie zwölf hätte sich mit den versteinerten Samen aus Kategorie zehn verbinden lassen. Auch hätten sich viele Objekte der Kategorien dreizehn bis fünfzehn unter dem Sammelbegriff „Artificialia“ zusammenfassen lassen, der im frühneuzeitlichen Sammlungswesen eine lange Tradition besaß und in der Regel genau solche Objekte umfasste.

Diese Unschärfe in der Klassifizierung der Objekte verstärkt den Eindruck, dass Müller sich nicht an der ursprünglichen Systematik von Johann Christoph Olearius orientierte und ihm auch kein Verzeichnis der vollständigen Sammlung vorlag, das er hätte zum Vorbild nehmen können. Denkt man an die beiden Verzeichnisse der Münzen zurück, die erst durch das Kaufinteresse Gottfried Bessels entstanden waren, so ist davon auszugehen, dass Johann Christoph Olearius kein Verzeichnis seiner Sammlung geführt hatte. Unter diesem Gesichtspunkt stellt sich die Frage, unter welchen Voraussetzungen Müller die Sammlung seines Großvaters rekonstruierte und ob er dabei auch auf schriftliche Dokumente zurückgreifen konnte. Dass er sein Verzeichnis durch persönliche Katalogisierungsarbeiten bei allen Verwandten erstellte, erscheint ausgeschlossen, da, wie die unrichtige Namenszuschreibung an seinen Onkel Georg Volkmar Hartmann zeigte, die Beziehungen innerhalb der Familie Olearius offenbar durchwachsen waren und Müller zudem nur inkonsequent auf die zeitgenössischen Provenienzen der Stücke einging. Der Großteil der Objekte wird ohne Besitzvermerk angegeben, was die Frage aufwirft, ob Müller die genaue Aufteilung der Objekte innerhalb der Familie überhaupt bekannt war.

Im gesamten Text mit seinen 15 Kategorien wird nur acht Mal auf den aktuellen Besitzer verwiesen. So war die Witwe Eleonore Sabine Olearius im Besitz der gesamten Kategorie Humana. Ihr Sohn Johann Christian besaß die Objekte der Kategorien Sacra und Vegetabilia sowie das in Kategorie 15 verzeichnete Brettspiel. Die Familie Schuhmann besaß einen Großteil der Objekte aus der Kategorie Marina. Laut Müller war diese Teilsammlung sehr gut geordnet und verfügte auch über einen Katalog. Darüber hinaus soll der Naturforscher Friedrich Christian Lesser (1692-1754) sie sehr bewundert haben. Müllers eigene Familie war im Besitz der Teilsammlungen Arma und Animalia sowie eines Teils der exotischen Gewänder und Kopfbedeckungen. Obwohl die Familie Hartmann von Müller am Ende seines Verzeichnisses ebenfalls als Besitzer zumindest eines Teils der Sammlung von Johann Christoph Olearius genannt wird, findet sich im gesamten Text kein Hinweis auf sie, weshalb unklar ist, ob die in ihrem Besitz befindlichen Objekte überhaupt Eingang in das Verzeichnis gefunden haben. 
Der von Müller zusammengestellte Katalog über die Sammlung seines Großvaters ist deshalb unter einigen Vorbehalten zu benutzen. Zum einen sollte kein Anspruch auf Vollständigkeit daran gestellt werden, da durch die inkonsequente Angabe von Provenienzen unklar ist, ob Müller alle noch vorhandenen Sammlungsteile verzeichnet hatte oder nur ausgewählte Teile, von denen er Kenntnis erlangt hatte. Zum anderen können die beschriebenen Objekte nicht mit einem älteren, vom Sammler selbst angefertigten Katalog verglichen werden, sodass ebenfalls unklar bleibt, ob in der Zeit vom Tod des Johann Christoph Olearius bis zur Publikation des Verzeichnisses durch seinen Enkel bereits Objekte veräußert worden waren. Wie die große Masse an Objekten ohne Provenienzangabe zu interpretieren ist, bleibt ebenfalls offen.

Neben dieser Vielzahl an Fragen, die das Verzeichnis von Müller eröffnet, trägt es aber gleichzeitig zur Klärung anderer Punkte bei. So lässt sich etwas über die Verteilung der Sammlung an die Erbengemeinschaft sagen, die von Johann Christoph Olearius offenbar nicht im Detail festgelegt worden war, denn an zwei Stellen verweist Müller auf ein Losverfahren, über das die Sammlung auf die einzelnen Familien aufgeteilt worden war. Im Provenienzvermerk zu den Kleidungsstücken und Utensilien, die seine Familie erhalten hatte, heißt es: Dieses alles ist auf das Müllerische Loos gekommen. ${ }^{79}$ Noch deutlicher wird der Zusatz zu den sakralen Objekten, die Johann Christian Olearius erhalten hatte: Diese Section hat im Loos des seel. Besitzers Sohn M. Io. Christian. Olearius, Diac. Arnstad. bekommen, welcher solche als ein Liebhaber von dergleichen Dingen sorgfältig aufbebet. $80 \mathrm{Da}$ bei den anderen sechs Provenienzvermerken wiederum kein Hinweis auf ein solches Losverfahren enthalten ist, muss offenbleiben, ob die gesamte Sammlung auf diese Weise aufgeteilt wurde.

Was nun den Zweck des von Müller erstellten Verzeichnisses betrifft, so kann mangels selbsterklärender Worte des Autors nur der Versuch einer Annäherung unternommen werden. Hier liefert das Titelblatt wertvolle Hinweise. Auf diesem bezeichnet sich Johann Gottfried Müller als Ehrenmitglied der Lateinischen Gesellschaft zu Jena. Diese Gelehrtengesellschaft war 1733 an der Universität Jena gegründet worden und hatte sich der Pflege und Übung der lateinischen Sprache verschrieben. Sie ging unmittelbar aus der 1728 an derselben Universität gegründeten Deutschen Gesellschaft zu Jena hervor, die sich - wie der Name bereits vermuten lässt - der deutschen Sprache widmete. ${ }^{81}$ Wichtiger als Müllers eigene

79 Ebd., S. 7.

80 Ebd., S. 5.

81 Vgl. Felicitas Marwinski, Johann Andreas Fabricius und die Jenaer Gelehrtengesellschaften des 18. Jahrhunderts, Jena 1989, S. 84-86; Jürgen OvERHoff, Die Frühgeschichte des Philantropismus (1715-1771). Konstitutionsbedingungen, Praxisfelder und Wirkung eines pädagogischen Reformprogramms im Zeitalter der Aufklärung (Hallesche Beiträge zur Europäischen Aufklärung 26), Tübingen 2004, S. 33; HolgER Zaunstöck, Sozietätslandschaft und Mitgliederstrukturen. Die mitteldeutschen Aufklärungsgesellschaften im 18. Jahrhundert (Hallesche Beiträge zur Europäischen Aufklärung 9), Tübingen 1999, S. 2, 95 f., 168, 185, 281. 
Zugehörigkeit zu einer der vielfältigen Sozietäten des 18. Jahrhunderts ist die Wahl seines Widmungsempfängers. Das Verzeichnis ist niemand Geringerem als Franz Ernst Brückmann (1697-1753) gewidmet, einem in Wolfenbüttel ansässigen Arzt und Naturforscher. Brückmann war Mitglied der Leopoldina zu Halle und der Preußischen Akademie der Wissenschaften zu Berlin und hatte zahlreiche Publikationen auf dem Gebiet der Mineralogie und des Bergbaus, sowie Bibliografien im Bereich Numismatik und Tierwelt veröffentlicht. ${ }^{82}$ Nicht ungewöhnlich, jedoch im Zusammenhang mit Müllers Verzeichnis erwähnenswert ist, dass Brückmann über ein umfangreiches Naturalienkabinett verfügte. Da Müller in den ersten zehn Kategorien seiner Auflistung gleich sieben versammelte, die eindeutig dem Sammlungsgebiet der „Naturalia“ zuzuordnen sind, ist die Frage zu diskutieren, ob dem Widmungsempfänger die ihm zugeeignete Sammlung mit dieser Publikation schmackhaft gemacht werden sollte.

Ob es im Nachgang zur Publikation von Müllers Verzeichnis zu einem Verkauf von weiteren Teilen der ehemaligen Sammlung Olearius gekommen ist, lässt sich nicht mehr feststellen. Ebenso wenig ist klar, was aus den Sammlungen im Besitz der einzelnen Erben geworden ist. Zahlreiche Fallbeispiele von Gelehrten und ihren Privatsammlungen zeigen, dass in den meisten Fällen die neuen Besitzer, sollten sie kein berufliches oder wissenschaftliches Interesse mit der Sammlung verbinden können, diese häufig sehr schnell zum Verkauf anboten. Im Fall der Sammlung Olearius lassen sich nur jene Objekte weiterverfolgen, die in den Besitz von Institutionen übergegangen sind, wie etwa die Lutherschriften in der Kirchenbibliothek zu Arnstadt oder die Münzen im Stift Göttweig.

Dass im Übrigen Münzen in keiner der von Müller erstellten Kategorien erwähnt werden, lässt darauf schließen, dass Johann Christoph Olearius tatsächlich seine gesamte Münzsammlung noch zu seinen Lebzeiten veräußerte. Ob aber die gesamte Sammlung an Gottfried Bessel verkauft wurde oder, wie bei Berghaus angegeben, die Goldmünzen über Umwege nach Dresden gelangt sind, lässt sich aus heutiger Sicht nicht mehr nachprüfen.

\section{Die Stiftssammlungen bis heute}

Ebenso wenig nachprüfen lässt sich, in welchem Ausmaß sich noch heute Münzen aus der Sammlung Olearius in Göttweig befinden. Dies liegt zum einen am Fehlen eines zeitgenössischen Katalogs, sowohl von der gesamten Sammlung des Johann Christoph Olearius als auch der nach Göttweig gelangten Stücke, sodass nicht gesichert ist, aus welchen Einzelstücken sich seine Münzsammlung zusammensetzte und welche Teile daraus von Gottfried Bessel angekauft wurden. War eine generische Zusammenstellung der Münzen nach Motiv, Metall und Stückzahl für

82 Vgl. Rudolph Zaunick, Brückmann, Franz Ernst, in: Neue Deutsche Biographie, Bd. 2, Berlin 1955, S. 655 f. 
die Verkaufsverhandlungen zwar ausreichend, so bedurfte die museale Aufbereitung und Einordnung innerhalb der Göttweiger Stiftssammlungen eines detaillierten Stückverzeichnisses. Dessen war sich auch Gottfried Bessel bewusst, weshalb die anfänglich zugesagte Verfügbarkeit des Johann Christian Olearius eine willkommene Gelegenheit zur Beseitigung dieses Mankos war. Obwohl Gottfried Bessel, vor allem in Zusammenhang mit seinen eigenen geschichts- und hilfswissenschaftlichen Studien, über einen Stab an entsprechend ausgebildeten Mitarbeitern verfügte, fehlte es offenbar an einem Gelehrten mit numismatischer Expertise. Nur so ist zu erklären, weshalb in Göttweig nicht nur eine Verzeichnung der angekauften Sammlung Olearius unterblieb, sondern darüber hinaus zu Lebzeiten Bessels überhaupt kein Katalog der dortigen Münzsammlung erstellt wurde. Erst der spätere Prior Rupert Krenner (1722-1782), der noch unter Bessel in Göttweig Profess abgelegt hatte, legte in seinen letzten Lebensjahren einen 225 Seiten starken Elenchus nummorum an, der jedoch nur seine eigene Privatsammlung umfasste. Diese dürfte jedoch nach seinem Tod in der Stiftssammlung aufgegangen sein. ${ }^{83}$

Erst mit der Wende zum 19. Jahrhundert sind in Göttweig eigene Kustoden für die Münzsammlung nachweisbar, die im Rahmen ihrer Tätigkeit entsprechende Verzeichnisse anlegten. Interessant ist das 1867 von Willibald Bobisch (1801-1873) angelegte Summarische Verzeichnis der Münz-Sammlung, bei dem es sich um einen als Standortverzeichnis geführten Katalog handelte. Daraus wird ersichtlich, dass die Münzsammlung von fünf Schränken unter Abt Bessel - wie auf dem Kupferstich Salomon Kleiners ersichtlich ist - auf mittlerweile sieben Schränke angewachsen war. Dass die von Bessel in Auftrag gegebenen Münzschränke noch in Verwendung waren, zeigt sich anhand der expliziten Nennung jenes u-förmigen Tisches mit Münzladen, der auch bei Kleiner zu sehen ist. ${ }^{84}$ Laut Bobisch bestand die Münzsammlung aus 17071 Münzen und Medaillen, von denen 357 Stück aus Gold, 6685 aus Silber und 10029 aus Kupfer, Zinn, Bronze und anderen Materialien bestanden. In den Münzschränken war der Bestand wie folgt aufgeteilt: Schrank I beherbergte Kupfermünzen, Medaillendubletten und Wappenabdrücke; Schrank II antike römische und byzantinische Münzen; Schrank III Münzen von römischen Konsuln und Kaisern, antike griechische Münzen aus Europa, Asien und Afrika; Schrank IV Medaillen aus diversen europäischen Ländern; Schrank V weitere Medaillen und Brakteaten; Schrank VI Münzen von Städten, Bistümern, Klöstern und Päpsten sowie Varia, und schließlich beherbergte der Tisch weitere Münzen von Päpsten, Kardinälen und Regenten des Hauses Lothringen. ${ }^{85}$ Anga-

83 Vgl. Göbl, Münzkabinett (wie Anm. 8), S. 162 f.; Lashofer, Profeßbuch (wie Anm. 25), S. 225; Lechner, Einleitung (wie Anm. 5), S. 22 f. (als Todesjahr Krenners wird jedoch irrig 1762 angegeben).

84 Vgl. Göвl, Münzkabinett (wie Anm. 8), S. 163; Lechner, Einleitung (wie Anm. 5), S. 24. Von den Münzschränken haben sich heute nur noch zwei erhalten, der Tisch ist nicht darunter.

85 Vgl. Lechner, Einleitung (wie Anm. 5), S. 24. 
ben zur Provenienz der Einzelstücke fehlen ebenso wie sich in der Sammlung selbst keine Unterlagszettel des 18. Jahrhunderts erhalten haben, mit deren Hilfe sich frühere Sammlungen rekonstruieren lassen könnten.

Die wechselvolle Geschichte des Stiftes Göttweig im 20. Jahrhundert macht es überdies schwierig, Stücke aus der älteren Göttweiger Münzsammlung heute noch nachzuweisen. Unter dem Vorwand der Misswirtschaft und der Konspiration gegen das nationalsozialistische Regime wurde das Stift im Jahr 1939 durch den Kremser Oberbürgermeister Franz Retter (1898-nach 1948) aufgelöst und unter die kommissarische Verwaltung der Stadt Krems gestellt. ${ }^{86}$ Schon zuvor hatte Retter versucht, an die Besitzungen des Klosters zu gelangen, indem er drohte, es gewaltsam auflösen zu lassen, sollte der Konvent es nicht freiwillig übergeben. Um sein Angebot attraktiver zu machen, bot Retter jedem Konventualen, der über kein Einkommen als Seelsorger verfügte, eine monatliche Leibrente an, wenn im Gegenzug sämtliche beweglichen und unbeweglichen Klostergüter der Stadt Krems übertragen würden. In dem Wissen, dass Vermögen, Sachen und Rechte nur durch staatliche Behörden eingezogen werden durften, lehnte der Konvent ab, woraufhin Retter die Geheime Staatspolizei einschaltete, die im Februar 1939 zu einer ersten Durchsuchung des Stiftes in Göttweig eintraf. Als Folge der eingeleiteten Untersuchungen wurden Abt Hartmann II. Strohsacker (1870-1946) und mehrere Koventualen verhaftet und nach Wien zu weiteren Verhören gebracht. Ein Verfahren zur Auflösung des Klosters wurde eingeleitet, nach geltender Rechtslage jedoch zu Gunsten des Landes Österreich. Franz Retter favorisierte aber weiterhin eine Enteignung Göttweigs zu Gunsten der Stadt Krems, weshalb er gegen den Willen des Reichskommissars für die Wiedervereinigung Österreichs mit dem Deutschen Reich, Josef Bürckel (1895-1944), persönlich beim Reichsminister des Innern, Wilhelm Frick (1877-1946), Einspruch erhob. Als Ergebnis dieser Intervention wurde erstmals die Erlaubnis erteilt, eine Enteignung zu Gunsten einer anderen Rechtsperson als des Landes Österreich durchzuführen. ${ }^{87}$

Beschwerden des apostolischen Nuntius Cesare Orsenigo (1873-1946) beim Auswärtigen Amt in Berlin oder des ehemaligen österreichischen Bundeskanzlers Rudolf Ramek (1881-1941), der Göttweig als Anwalt vertrat, beim österreichischen Bundesgerichtshof blieben ohne Erfolg und so wurde Göttweig am 1. August 1939 als erstes großes Stift in Österreich aufgelöst. Über 30 weitere sollten folgen. 88

Sämtliche Konventualen wurden aus dem Stift vertrieben. Während die jüngeren zur Wehrmacht eingezogen wurden, wurden andere als Seelsorger auf die

86 Vgl. Helmut Engelbrecht, Göttweig zur Zeit der Ersten Republik und der NS-Herrschaft, in: Studien und Mitteilungen zur Geschichte des Benediktinerordens und seiner Zweige 94 (1983): Geschichte des Stiftes Göttweig 1083-1983. Festschrift zum 900-Jahr-Jubiläum, S. 386-429, hier S. 417 f.; SPEvaK, Kunst (wie Anm. 7), S. 143 f.

87 Vgl. Engelbrecht, NS-Herrschaft (wie Anm. 86), S. 418-421; SpevaK, Kunst (wie Anm. 7), S. 143.

88 Vgl. SpevaK, Kunst (wie Anm. 7), S. 143. 
(ehemaligen) Stiftspfarren verteilt. Abt Strohsacker und die älteren Konventualen wurden in das Propsteihaus in Unternalb verbracht. Die Liegenschaften des Stiftes gingen in Kremser Eigenverwaltung über und wurden in weiterer Folge zum Teil verkauft. 1940 wurde mit dem Abtransport der Mobilien aus Göttweig begonnen. Die von Gottfried Bessel eingerichteten Sammlungen, mittelalterliche Handschriften, Bücher und Porzellan wurden nach Krems verbracht, wo sie im Museum der Stadt ausgestellt wurden. Die verbliebenen Teile der Stiftsbibliothek, das Stiftsarchiv, das Musikarchiv, ein Teil der Gemäldesammlung sowie die Einrichtung der Kaiserzimmer wurden vorerst im Stift belassen. Das übrige Mobiliar wurde im Kremser Rathaus zur Benutzung aufgestellt beziehungsweise in die Minoritenkirche zu Stein ins Depot gebracht. Vier LKW-Ladungen an Möbeln wurden nach Wien an die Gauleitung von Niederdonau geliefert. Die von Gottfried Bessel eingerichtete Waffensammlung wurde dem Gauleiter Hugo Jury (1887-1945) zum Geschenk gemacht. ${ }^{89}$

Mit der Bombardierung von Krems wurden die Sammlungen in Zusammenarbeit mit dem Amt für Denkmalpflege (später: Bundesdenkmalamt) in von diesem eingerichtete Bergungsorte verbracht. So gelangte das Musikarchiv in das Stift Altenburg, die Münzen in das Stift St. Florian ${ }^{90}$ und die ausgelagerten Gemälde sowie wertvolle Kunstobjekte nach Bad Aussee. Eine nicht fassbare Anzahl an Büchern und Kunstobjekten wurde über das Wiener Auktionshaus Dorotheum verkauft, da die Stadt Krems mit der Übernahme der Göttweiger Besitzungen auch die finanziellen Verpflichtungen des Stiftes übernommen hatte, sodass die so hartnäckig angestrebte Enteignung kaum Gewinn brachte. Das Stift selbst wurde nach verschiedenen Zwischennutzungen der "Gesellschaft zur Förderung und Pflege deutscher Kulturdenkmäler“ übergeben, die darin eine „Nationalpolitische Erziehungsanstalt" einrichtete.91

Nach Kriegsende wurde das Stift durch den neuen provisorischen Bürgermeister der Stadt Krems, Karl Suppanz (1879-1958), dem Konvent restituiert und dessen verbliebenen Mitgliedern die Rückkehr ermöglicht. Abt Strohsacker sowie seine Nachfolger Edmund Vašiček (1884-1949) und Wilhelm Zedinek (1898-1971) bemühten sich um eine vollständige Restitution der Mobilien und Immobilien, sodass noch 1945 ein LKW mit Möbeln aus Krems zurückgebracht wurde. Für die Restitution der Kunstsammlungen erwies es sich als hilfreich, dass der Verwalter des Stadtmuseums Krems, Hans Plöckinger (1882-1955), diese bei der Übernahme

89 Vgl. Engelbrecht, NS-Herrschaft (wie Anm. 86), S. 425; Spevak, Kunst (wie Anm. 7), S. $145-147$.

$90 \mathrm{Zu}$ einem ungeklärten Zeitpunkt wurden die Goldmünzen vom Rest der Sammlung getrennt. Sie sind heute nicht mehr auffindbar. Ob die Vermutung von Gregor Martin Lechner, die Reichsbank habe die Goldmünzen der Göttweiger Münzsammlung konfisziert, zutrifft, ist zu diskutieren: vgl. LeCHNER, Einleitung (wie Anm. 5), S. 24.

91 Vgl. Engelbrecht, NS-Herrschaft (wie Anm. 86), S. 428; SPevak, Kunst (wie Anm. 7), S. $148 \mathrm{f}$. 
1939 inventarisiert hatte, sodass ein Großteil der Sammlungen noch auffindbar war.

Die dem Kremser Stadtarchiv einverleibte Graphische Sammlung wurde am 21. Oktober 1947 restituiert. Bei einer 1956 durchgeführten Inventur stellte sich jedoch heraus, dass zwölf Boxen mit insgesamt 482 Blättern fehlten. Aus der Münzsammlung war die numismatische Spezialliteratur vom Kremser Stadtmuseum an das Bundesdenkmalamt übergeben worden, das die Bücher nach Bad Aussee verbracht hatte. Von dort wurden sie nach Kriegsende von US-Behörden nach München gebracht, ehe sie über Salzburg und St. Florian nach Göttweig zurückkehrten. Die Münzkästen waren nach Schloss Ennsegg und in das Stift Hohenfurth ausgelagert worden. Die Münzen waren teils nach St. Florian und teils nach Kremsmünster gebracht worden, die Brakteatensammlung konnte in Wien ausfindig gemacht werden. Jener Teil der Gemäldesammlung, der im Stift verblieben war, war großteils zerstört. Die übrigen Gemälde waren in Bergungsorten des Bundesdenkmalamtes und in Privatwohnungen untergebracht worden, von wo sie 1950 nach Restaurierungsarbeiten durch das Bundesdenkmalamt zurückgestellt wurden. Noch 1947 wurden die Inkunabeln aus dem Kremser Stadtarchiv zurückgestellt. Die mittelalterlichen Handschriften waren durch das Bundesdenkmalamt nach Bad Aussee und in das Stift Seitenstetten verbracht worden, von wo aus sie 1948 restituiert wurden. Von der Gauleiter Jury geschenkten Waffensammlung ließen sich noch 73 Objekte ausfindig machen, die am 17. Oktober 1950 restituiert wurden. Die Antikensammlung wurde nur fragmentarisch vom Kremser Stadtmuseum zurückgegeben. Was aus den fehlenden Objekten geworden ist, ist unklar. Von den bis 1945 verkauften Gegenständen aus Göttweiger Besitz konnten nur wenige bis 1958 wiedergefunden werden. Dass die Stadt Krems mit den Nachforschungen zu diesen Gegenständen Personen beauftragt hat, die an deren Enteignung beteiligt gewesen waren, wie etwa Hans Plöckinger, mutet aus heutiger Sicht befremdlich an. ${ }^{92}$

Weitaus schwieriger als die Restitution der Kunstgegenstände verlief jene der Liegenschaften und der daraus gewonnenen Erträge. Auf Grundlage der von 1946 bis 1949 erlassenen Rückstellungsgesetze verklagte das Stift die Stadt Krems auf Herausgabe der in Kremser Besitz befindlichen Liegenschaften, auf Rückstellung sämtlicher aus Klostergütern gewonnenen Erträge sowie auf Schadenersatz für abhanden gekommene Vermögenswerte. Da die Stadt hochverschuldet war und die vom Stift verlangten Entschädigungen nicht zahlen konnte, einigte man sich in allen Fällen außergerichtlich, wobei die letztlich gezahlten Summen meist weit unter den ursprünglichen Forderungen des Stiftes lagen. ${ }^{93}$

92 Vgl. Engelbrecht, NS-Herrschaft (wie Anm. 86), S. 429; Clemens Lashofer, Jüngste Vergangenheit und Gegenwart, in: Geschichte des Stiftes Göttweig (wie Anm. 86), S. 430-451, hier S. 439 f.; SpevaK, Kunst (wie Anm. 7), S. 150-153.

93 Vgl. Lashofer, Vergangenheit (wie Anm. 92), S. 441-445; SpevaK, Kunst (wie Anm. 7), S. $154 \mathrm{f}$. 
In den Nachkriegsjahren lag der Fokus des Stiftes vor allem auf der wirtschaftlichen Stabilisierung und der quantitativen Restitution seines Besitzes, weshalb die Kunstsammlungen erst mit jahrelanger Verzögerung wieder eingerichtet und dabei neu verzeichnet werden konnten. Dadurch wurde das Fehlen mancher Objekte oder gar ganzer Objektgruppen erst spät entdeckt. Fehlende Vergleichsdokumente lassen fallweise keine Aussage zu, welche Stücke bis heute verschollen sind.

Bezogen auf das von Gottfried Bessel eingerichtete Münzkabinett ergibt sich die Situation, dass aufgrund bereits fehlender zeitgenössischer Kataloge einerseits und einem aktuellen physisch dezimierten Bestand andererseits keine Aussage darüber getroffen werden kann, wie viele der von Bessel angekauften Münzen sich noch in der heutigen Münzsammlung befinden und um welche Stücke es sich handelt. 